\title{
DESIGN AND SYNTHESIS OF SOME SUBSTITUTED ACRIDINE DERIVATIVES OF ANTICIPATED ANTITUMOR ACTIVITY
}

S. M. El-Moghazy Aly ${ }^{1}$, D. E. Abdel Rahman ${ }^{1}$, S. E.-S. Abbas ${ }^{1}$, M. A.A. Mohammed ${ }^{1}$ and A. H. Amin ${ }^{2}$

${ }^{1}$ Department of Pharmaceutical Chemistry, Faculty of Pharmacy, Cairo University, Cairo-11562, Egypt

${ }^{2}$ Department of Pharmaceutical Chemistry, Faculty of Pharmacy, Beni Sueif University, Egypt

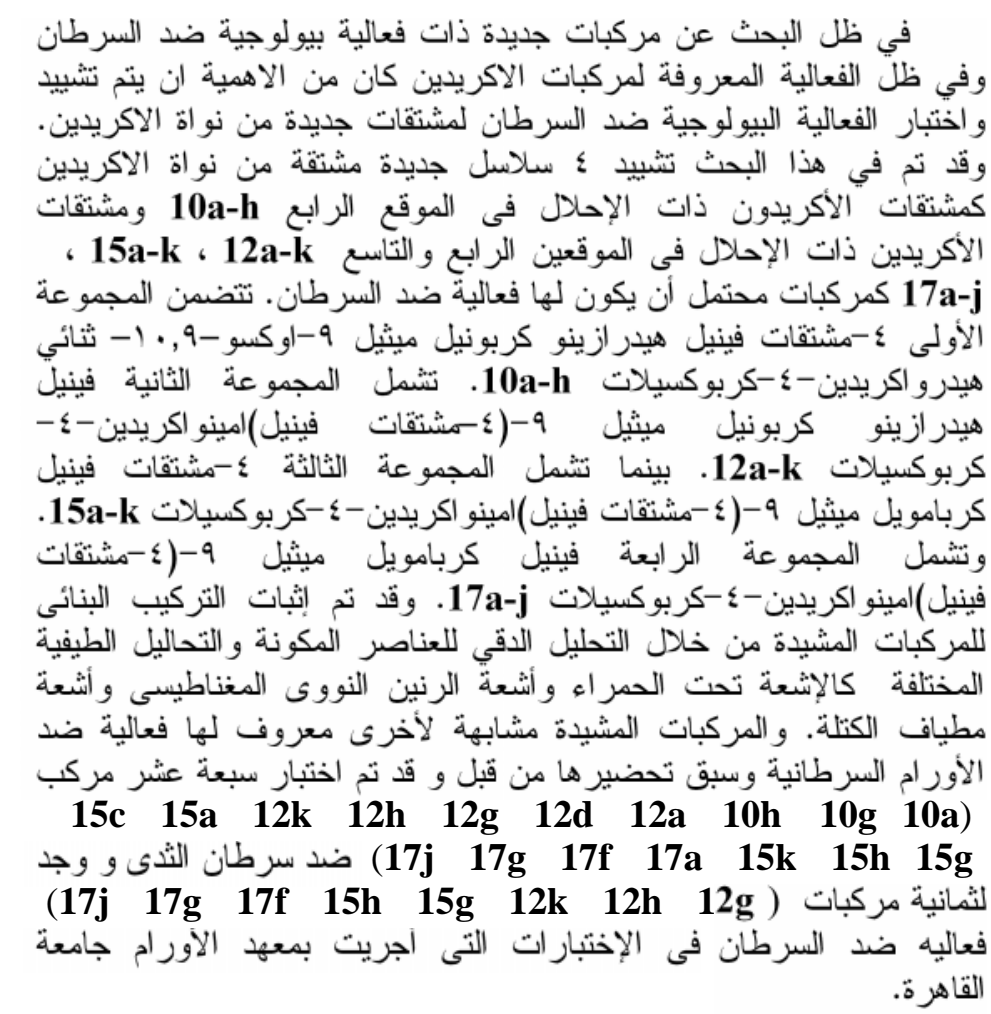

Four series of new acridine derivatives of anticipated antitumor activity have been designed and synthesized. The first series belongs to 4-substituted phenylhydrazinocarbonylmethyl 9-oxo9,10-dihydroacridine-4-carboxylate 10a-h. The second series 
consists of phenylhydrazinocarbonylmethyl 9-(4-substituted phenyl)aminoacridine-4-carboxylate $12 a-k$, while the third series comprises 4-substituted phenylcarbamoylmethyl 9-(4-substituted phenyl)aminoacridine-4-carboxylate $15 \boldsymbol{a}-\boldsymbol{k}$. The fourth one belongs to phenylcarbamoylmethyl 9-(4-substituted phenyl)aminoacridine4- carboxylate 17a-j. The chemical structure of synthesized compounds was elucidated by spectral data and elemental analysis. Seventeen selected compounds $(10 \mathrm{a}, 10 \mathrm{~g}, 10 \mathrm{~h}, 12 \mathrm{a}, 12 \mathrm{~d}, 12 \mathrm{~g}, 12 \mathrm{~h}$, $12 \mathrm{k}, 15 \mathrm{a}, 15 \mathrm{c}, 15 \mathrm{~g}, 15 \mathrm{~h}, 15 \mathrm{k}, 17 \mathrm{a}, 17 \mathrm{f}, 17 \mathrm{~g}$ and $17 \mathrm{j})$ were tested against breast cancer cell line (MCF7) and eight compounds (12g, $12 \mathrm{~h}, 12 \mathrm{k}, 15 \mathrm{~g}, 15 \mathrm{~h}, 17 \mathrm{f}, 17 \mathrm{~g}, 17 \mathrm{j})$ were found to exhibit significant antitumor activity.

\section{INTRODUCTION}

The most important class of antitumor drugs is the DNA intercalating agents. The majority of DNA intercalating antitumor drugs has a common general structure, comprising a tri or tetracyclic chromophore to which is attached one or two flexible side chains bearing cationic charges ${ }^{1}$. Acridines represent one of the early and most thoroughly investigated compounds.

Recently, series of $\mathrm{N}$-mustard derivatives of 9-anilinoacridine was synthesized and evaluated for cytotoxicity against human lymphoplastic leukemic cells (CCRFCEM) in culture. The results showed that, all of the new N-mustard derivatives exhibited significant cytotoxic activity ${ }^{2 \& 3}$. Furthermore, several mono and dinuclear isoquinolino[4,5-bc]acridine derivatives were designed and synthesized. Their DNA binding properties and antitumor activities were investigated and some of these compounds were found to be cytotoxic against P388 and A549 ${ }^{4 \& 5}$.
Moreover, a series of Nsubstituted triamine linked acridine dimers was synthesized and tested for cytotoxic activity. Most acridine dimer derivatives revealed high potent in vitro cytotoxicity properties and DNA binding activity ${ }^{6}$. Telomere and telomerase are attractive targets for anticancer therapeutic ${ }^{7}$. Replacement of aniline substituent in BRACO-19, a potent G-quadreplex binding molecule and telomerase inhibitor, by benzylamino- groups resulted in enhanced quadreplex interaction $^{8}$.

The carbonyl containing chromophore I, although it is planar, it binds significantly less tightly, but appears to intercalate DNA. Results of in vitro cytotoxicity revealed that compound I showed potential interesting levels of cytotoxicity ${ }^{9}$ Furthermore, in our laboratories compound II was prepared in analogy to compound I substituting alkyl side chain amide by an aromatic one and was found to show antitumor activity ${ }^{10}$. 
<smiles>CNCCOC(=O)c1cccc2c(=O)c3ccccc3[nH]c12</smiles>

I<smiles>N=C(N)NOS(=O)(=O)c1ccc(NC(=O)C2=CC=CC3C(=O)c4ccccc4NC23)cc1</smiles>

II

In our work, substitution of the carboxamide moiety of the acridone II by phenylhydrazinocarbonylmethyl carboxylate afforded $\mathbf{1 0 .}$

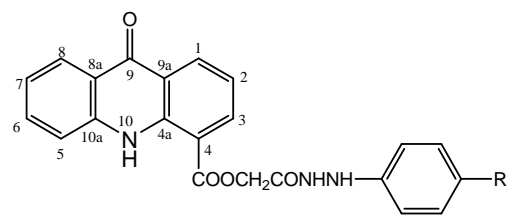

10

$\mathrm{R}=\mathrm{H}, \mathrm{CH}_{3}, \mathrm{Br}, \mathrm{OCH}_{3}, \mathrm{Cl}, \mathrm{COOH}, \mathrm{NO}_{2}$ and $\mathrm{SO}_{2} \mathrm{NH}_{2}$

Also, it was documented that N[2-(dimethylamino)ethyl]acridine-4carboxamide, DACA III showed a broad spectrum of in vivo activity against leukemia and solid tumors ${ }^{9}$. DACA III is known to be mixed topoisomerase I / II inhibitor ${ }^{11}$. In addition, compounds IV that were prepared in our laboratories, showed an interesting level of antitumor $\operatorname{activity}^{12}$.

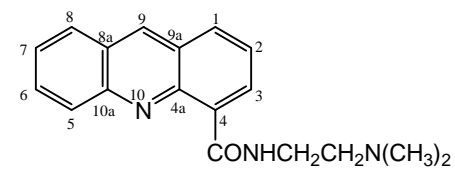

III<smiles>O=S(=O)(O)c1ccc(Nc2c3ccccc3nc3ccccc23)cc1</smiles>

IV

$\mathrm{R}=\mathrm{H}$, amidino and different heterocycles

Accordingly, in the present work, compounds 12, 15 and 17 are prepared as a structure hybrid of compounds III and IV by combining the characteristic feature of compound III having carbonyl function at position 4 of the acridine structure and the alignment of 9-anilino moiety in compound IV which is important in insertion into the minor groove.<smiles>O=COC(=O)c1ccc2c3c(c4ccccc4nc13)Nc1ccc(P)cc1-2</smiles>

$12 \mathrm{R}=\mathrm{H}, \mathrm{CH}_{3}, \mathrm{OCH}_{3}, \mathrm{Cl}, \mathrm{COOH}, \mathrm{NO}_{2}$ and $\mathrm{SO}_{2} \mathrm{NH}_{2}, \mathrm{SO}_{2} \mathrm{NHC}(\mathrm{NH}) \mathrm{NH}_{2}$, $\mathrm{SO}_{2} \mathrm{NHR}^{\prime}\left(\mathrm{R}^{\prime}=\right.$ different heterocycles)

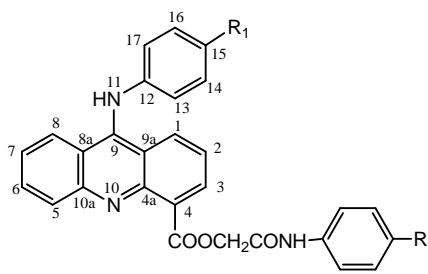

$15 \mathrm{R}=\mathrm{R}_{1}=\mathrm{H}, \mathrm{CH}_{3}, \mathrm{OCH}_{3}, \mathrm{Cl}, \mathrm{COOH}, \mathrm{NO}_{2}$ and $\mathrm{SO}_{2} \mathrm{NH}_{2}, \mathrm{SO}_{2} \mathrm{NHC}(\mathrm{NH}) \mathrm{NH}_{2}$ $\mathrm{SO}_{2} \mathrm{NHR}^{\prime}\left(\mathrm{R}^{\prime}=\right.$ different heterocycles $)$

$17 \mathrm{R}=\mathrm{H}, \mathrm{R}_{1}=\mathrm{CH}_{3}, \mathrm{OCH}_{3}, \mathrm{Cl}, \mathrm{COOH}, \mathrm{NO}_{2}$ and $\mathrm{SO}_{2} \mathrm{NH}_{2}, \mathrm{SO}_{2} \mathrm{NHC}(\mathrm{NH}) \mathrm{NH}_{2}$, $\mathrm{SO}_{2} \mathrm{NHR}^{\prime}\left(\mathrm{R}^{\prime}=\right.$ different heterocycles $)$ 


\section{EXPERIMENTAL}

\section{Torsion angle}

A comparative measurement of torsion angles (10, 4a, 4, CO) and torsion angle $(10 a, 8 a, 9,9 a)$ of the known active compounds I, II and that of the prepared compounds $\mathbf{1 0 h}$ (Table 1), and torsion angles $(10,4 \mathrm{a}$, $4, \mathrm{CO})$, torsion angle $(9,11,12,13)$ and torsion angle $(9 \mathrm{a}, 9,11,12)$ of the well known active compounds III and IV and that of the prepared compounds 12g, 15g and 17f (Table 2) revealed certain $3 D$ structural similarities. A comparison of the electrostatic potentials of these compounds have been constructed and presented, where certain similarities of these maps of the active compounds and the corresponding designed and prepared compounds were shown. For sake of torsion angle study HyperChem software ${ }^{13}$ had been used.

Table 1: Torsion angle results of compounds I, II and $\mathbf{1 0 h}$.

\begin{tabular}{|l|c|c|c|}
\hline & compounds I & compounds II & compounds 10h \\
\hline Torsion angle 10a,8a,9,9a & $11.2047^{\circ}$ & $18.7147^{\circ}$ & $14.5483^{\circ}$ \\
\hline Torsion angle 10,4a,4,CO & $1.91655^{\circ}$ & $3.97261^{\circ}$ & $1.59358^{\circ}$ \\
\hline
\end{tabular}

Table 2: Torsion angle results of compounds III, IV, 12g, 15g and 17f.

\begin{tabular}{|c|c|c|c|c|c||}
\hline & $\begin{array}{c}\text { compounds } \\
\text { III }\end{array}$ & $\begin{array}{c}\text { Compounds } \\
\text { IV }\end{array}$ & $\begin{array}{c}\text { compounds } \\
\mathbf{1 2 g}\end{array}$ & $\begin{array}{c}\text { compounds } \\
\mathbf{1 5 g}\end{array}$ & $\begin{array}{c}\text { compounds } \\
\mathbf{1 7 f}\end{array}$ \\
\hline Torsion angle 10,4a,4,CO & $2.77241^{\circ}$ & - & $5.20834^{\circ}$ & $3.96954^{\circ}$ & $2.9716^{\circ}$ \\
\hline Torsion angle 9, 11,12,13 & - & $18.0039^{\circ}$ & $25.4523^{\circ}$ & $22.1519^{\circ}$ & $22.7416^{\circ}$ \\
\hline Torsion angle 9a, 9, 11,12 & - & $85.0268^{\circ}$ & $72.5832^{\circ}$ & $78.2066^{\circ}$ & $77.7536^{\circ}$ \\
\hline
\end{tabular}

\section{Procedure}

First the structure models were generated, then fully minimized to obtain the optimum structures using RMS gradient of 0.1 and FletcherReeves algorism. The electrostatic potential maps were constructed first by calculating the charges by Modified Neglect of Differential Overlap (MNDO) method. The potential maps were calculated, derived from a single point charge models at horizontal grid points $=100$ and vertical grid points $=100$ and 
contour level of 50 and an increment of 0.1 . The obtained isopotential surfaces were moved in space together with the underlying molecules in order to obtain maximum overlap.

\section{Chemistry}

Melting points were determined by open capillary tube using IA 9100 MK/ Digital Melting Point Apparatus and were uncorrected. IR Spectra were recorded as $\mathrm{KBr}$ pellets on Bruker Vector 22. ${ }^{1} \mathrm{H}$ NMR spectra were recorded on Jeol FT 90Q, 300 $\mathrm{MHz}$ using DMSO- $\mathrm{d}_{6}$ as solvent and were reported as $\delta$ values in ppm relative to tetramethylsilane (TMS) as internal standard. Electron Impact at 70 ev were performed with Finnegan MAT, SSQ 7000, Mass Spectrometers. Elemental analyses were carried out at the Microanalyical
Center, Faculty of Science, Cairo University and National Research Center.

Syntheses of intermediate compounds $\mathbf{3 b}-\mathbf{h}^{14-17}, \mathbf{4 a - c ^ { 1 8 }}, \mathbf{7}^{19}$, $\mathbf{8}^{19 \& 20}$, and $9^{19}$ were according to the literature.

Chloroacetic acid, 2-(substituted phenyl)hydrazide 4d-h (Scheme 1, Table 3)

4-Substituted phenylhydrazine 3dh (12 mmol) was dissolved in the least amount of dry benzene, followed by dropwise addition of chloroacetyl chloride $(2.5 \mathrm{ml}, 15 \mathrm{mmol})$ under stirring at room temperature. The reaction mixture was stirred for further $10 \mathrm{~min}$ then ice-cold water (50 $\mathrm{ml}$ ) was added while stirring. The obtained precipitate was filtered and crystallized from ethanol.

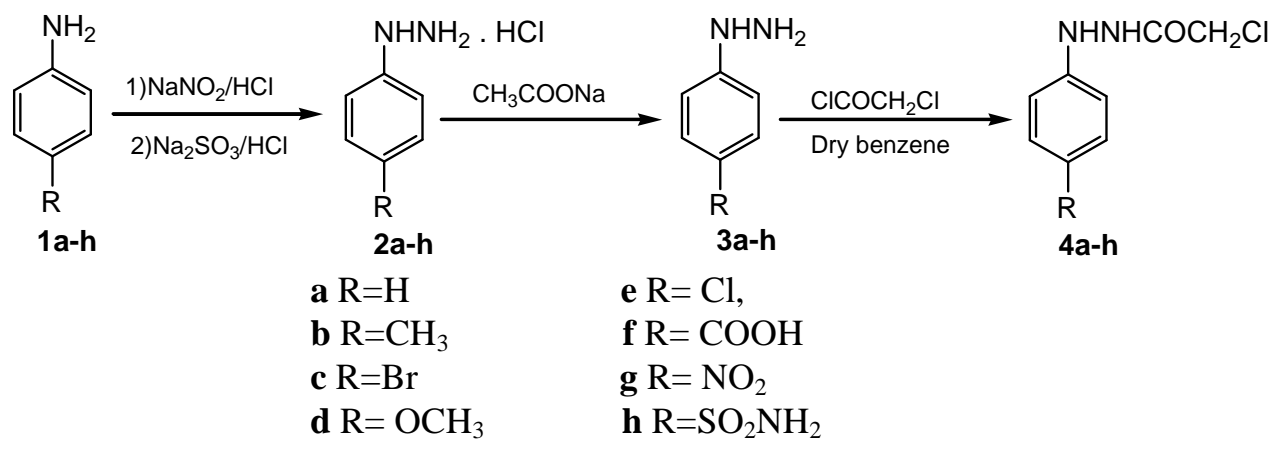

Scheme 1 
Table 3: Physical, analytical and IR spectral data for compounds 4d-h.

\begin{tabular}{|c|c|c|c|c|c|c|}
\hline \multirow{2}{*}{$\begin{array}{l}\text { Cpd. } \\
\text { No. }\end{array}$} & \multirow{2}{*}{$\mathrm{R}$} & \multirow{2}{*}{$\begin{array}{l}\text { Mol. Formula } \\
\text { Mol. Wt. }\end{array}$} & \multirow{2}{*}{$\begin{array}{l}\text { m.p }{ }^{\circ} \\
\text { Yield \% }\end{array}$} & \multicolumn{2}{|c|}{ Micro analysis } & \multirow{2}{*}{$\mathrm{IR} \mathrm{cm}^{-1}$} \\
\hline & & & & Calc. & Found & \\
\hline 4d & $\mathrm{OCH}_{3}$ & $\begin{array}{c}\mathrm{C}_{9} \mathrm{H}_{11} \mathrm{ClN}_{2} \mathrm{O}_{2} \\
214.65\end{array}$ & $\begin{array}{c}221-3 \\
54\end{array}$ & $\begin{array}{lr}\text { C } 50.36 \\
\mathrm{H} & 5.17 \\
\mathrm{~N} & 13.05 \\
\end{array}$ & $\begin{array}{l}\text { C } 50.74 \\
\mathrm{H} \quad 5.60 \\
\mathrm{~N} 13.09 \\
\end{array}$ & $\begin{array}{l}\text { 3446br }(2 \mathrm{NH}), 3068(\mathrm{CH} \text { arom. }), \\
2964,2840\left(\mathrm{CH}_{3}, \mathrm{CH}_{2}\right), 1652(\mathrm{CO}), \\
1604,1541,1511(\mathrm{NH}, \mathrm{C}=\mathrm{C}) .\end{array}$ \\
\hline $4 e$ & $\mathrm{Cl}$ & $\begin{array}{c}\mathrm{C}_{8} \mathrm{H}_{8} \mathrm{Cl}_{2} \mathrm{~N}_{2} \mathrm{O} \\
219.07\end{array}$ & $\begin{array}{c}192-3 \\
68\end{array}$ & $\begin{array}{l}\text { C } 43.86 \\
\text { H } 3.68 \\
\text { N } 12.79\end{array}$ & $\begin{array}{l}\text { C } 43.70 \\
\text { H } 3.90 \\
\text { N } 12.81\end{array}$ & $\begin{array}{l}3422,3206(2 \mathrm{NH}), 2986,2929\left(\mathrm{CH}_{2}\right), \\
1681(\mathrm{CO}), 1652,1584,1494(\mathrm{NH}, \\
\mathrm{C}=\mathrm{C}) .\end{array}$ \\
\hline $4 f$ & $\mathrm{COOH}$ & $\begin{array}{c}\mathrm{C}_{9} \mathrm{H}_{9} \mathrm{ClN}_{2} \mathrm{O}_{3} \\
228.64\end{array}$ & $\begin{array}{c}196-8 \\
81\end{array}$ & $\begin{array}{lr}\mathrm{C} & 47.28 \\
\mathrm{H} & 3.97 \\
\mathrm{~N} & 12.25 \\
\end{array}$ & $\begin{array}{l}\mathrm{C} 47.32 \\
\mathrm{H} \quad 3.50 \\
\mathrm{~N} 11.98 \\
\end{array}$ & $\begin{array}{l}\text { 3353,3253(2NH),3023(CHarom.), } \\
\text { 2668,2551(OHacid),1672(2CO), } \\
\text { 1605, 1538, } 1514(\mathrm{NH}, \mathrm{C}=\mathrm{C}) .\end{array}$ \\
\hline $4 \mathrm{~g}$ & $\mathrm{NO}_{2}$ & $\begin{array}{c}\mathrm{C}_{8} \mathrm{H}_{8} \mathrm{ClN}_{3} \mathrm{O}_{3} \\
229.62\end{array}$ & $\begin{array}{c}170-2 \\
77\end{array}$ & $\begin{array}{lc}\text { C } & 41.85 \\
\mathrm{H} & 3.51 \\
\mathrm{~N} & 18.3 \\
\end{array}$ & $\begin{array}{lr}\mathrm{C} & 41.76 \\
\mathrm{H} & 3.63 \\
\mathrm{~N} & 18.13 \\
\end{array}$ & $\begin{array}{l}3292(2 \mathrm{NH}), 2958,2925\left(\mathrm{CH}_{2}\right), 1662 \\
(\mathrm{CO}), 1596,1507(\mathrm{NH}, \mathrm{C}=\mathrm{C}) .\end{array}$ \\
\hline $4 h$ & $\mathrm{SO}_{2} \mathrm{NH}_{2}$ & $\begin{array}{c}\mathrm{C}_{8} \mathrm{H}_{10} \mathrm{ClN}_{3} \mathrm{O}_{3} \mathrm{~S} \\
263.71\end{array}$ & $\begin{array}{c}203-5 \\
82\end{array}$ & $\begin{array}{l}\text { C } 36.44 \\
\text { H } 3.82 \\
\text { N } 15.93\end{array}$ & $\begin{array}{l}\text { C } 36.76 \\
\text { H } 3.96 \\
\text { N } 15.69\end{array}$ & $\begin{array}{l}3349,3292,3186\left(\mathrm{NH}_{2}, 2 \mathrm{NH}\right), 3093 \\
(\mathrm{CHarom} .), 2960\left(\mathrm{CH}_{2}\right), 1653(\mathrm{CO}), \\
\text { 1605,1551(NH, C=C), 1294, } 1157 \\
\left(\mathrm{SO}_{2}\right) .\end{array}$ \\
\hline
\end{tabular}

4-Substituted phenylhydrazinocarbonylmethyl 9-oxo-9,10-dihydroacridine-4-carboxylate 10a-h (Scheme 2, Table 4)

Acridone-4-carboxylic acid $\mathbf{8}$ $(0.57 \mathrm{~g}, 2.4 \mathrm{mmol})$ was dissolved in 2 $\% \mathrm{NaOH}$ solution $(5 \mathrm{ml})$, followed by addition of ethanol $(40 \mathrm{ml})$. The resulting solution was evaporated to dryness and the yellow residue of sodium 9-oxo-9,10-dihydroacridine4-carboxylate 9 obtained was dried, then dissolved in DMF $(10 \mathrm{ml})$ and treated with an equimolar amount of 4a-h dissolved in DMF (10 ml). The reaction mixture was refluxed for 2 hrs, where $\mathrm{NaCl}$ was separated and filtered. The filtrate was poured onto ice-water $(150 \mathrm{ml})$. The precipitate formed was filtered, washed with water, dried and crystallized from DMF/water.

10a ${ }^{1} \mathrm{H}$ NMR: $\delta=5.11(\mathrm{~s}, 2 \mathrm{H}$, $\left.\mathrm{CH}_{2}\right), 7.08-8.59$ (m, $13 \mathrm{H}$, arom. $\underline{\mathrm{H}}$,

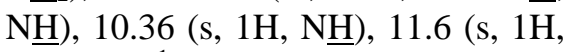
$\mathrm{NH}$ ). 10f ${ }^{1} \mathrm{H}$ NMR: $\delta=5.13(\mathrm{~s}, 2 \mathrm{H}$, $\left.\mathrm{C}_{2}\right), 7.31-8.54(\mathrm{~m}, 12 \mathrm{H}$, arom. $\underline{\mathrm{H}}$, $\mathrm{N} \underline{\mathrm{H}}), 10.4(\mathrm{~s}, 1 \mathrm{H}, \mathrm{N} \underline{\mathrm{H}}), 11.6(\mathrm{~s}, 1 \mathrm{H}$, $\mathrm{N} \underline{\mathrm{H}}), 11.97(\mathrm{~s}, 1 \mathrm{H}, \mathrm{COO} \underline{\mathrm{H}}) .10 \mathrm{a}$ MS: $\mathrm{m} / \mathrm{z}(\%) \mathrm{M}^{+}, 387,(0.36)$ and 221 , (100). 10g MS: m/z (\%) $\mathrm{M}^{+}, 432$ $(0.23)$ and $221,(100)$. 


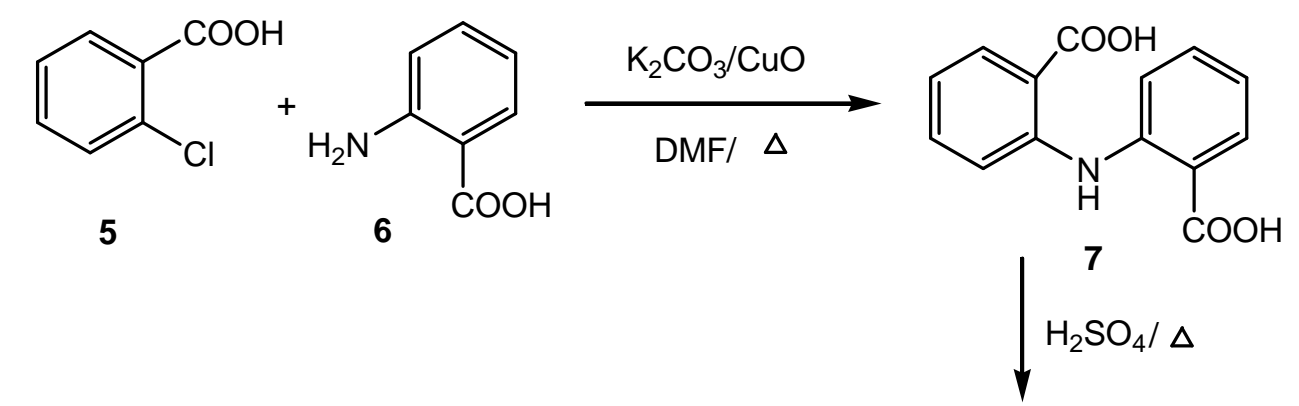<smiles>NC(=O)c1cccc2c(=O)c3ccccc3[nH]c12</smiles><smiles>O=C(O)c1cccc2c(=O)c3ccccc3[nH]c12</smiles>

9

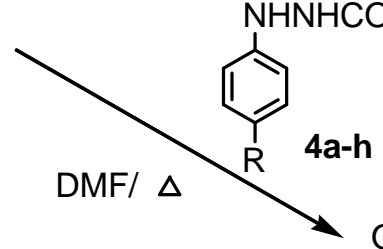<smiles>O=C(O)c1cccc2c(=O)c3ccccc3[nH]c12</smiles>

10a-h

$\mathrm{R}=\mathrm{H}, \mathrm{CH}_{3}, \mathrm{Br}, \mathrm{OCH}_{3}, \mathrm{Cl}, \mathrm{NO}_{2}, \mathrm{COOH}, \mathrm{SO}_{2} \mathrm{NH}_{2}$

\section{Scheme 2}


Table 4: Physical, analytical and IR spectral data for compounds 10a-h.

\begin{tabular}{|c|c|c|c|c|c|c|}
\hline \multirow{2}{*}{$\begin{array}{l}\text { Cpd. } \\
\text { No. }\end{array}$} & \multirow{2}{*}{$\mathrm{R}$} & \multirow{2}{*}{$\begin{array}{l}\text { Mol. Formula } \\
\text { Mol. Wt. }\end{array}$} & \multirow{2}{*}{$\begin{array}{l}\text { m.p }{ }^{\circ} \\
\text { Yield \% }\end{array}$} & \multicolumn{2}{|c|}{ Micro analysis } & \multirow{2}{*}{ IR $\mathrm{cm}^{-1}$} \\
\hline & & & & Calc. & Found & \\
\hline $10 \mathbf{a}$ & $\mathrm{H}$ & $\begin{array}{c}\mathrm{C}_{22} \mathrm{H}_{17} \mathrm{~N}_{3} \mathrm{O}_{4} \\
387.4\end{array}$ & $\begin{array}{c}259-61 \\
92\end{array}$ & $\begin{array}{lr}\mathrm{C} & 68.21 \\
\mathrm{H} & 4.42 \\
\mathrm{~N} & 10.85 \\
\end{array}$ & $\begin{array}{l}\mathrm{C} 68.27 \\
\mathrm{H} \quad 4.50 \\
\mathrm{~N} 10.45 \\
\end{array}$ & $\begin{array}{l}3420,3277(3 \mathrm{NH}), 3064(\mathrm{CH} \\
\text { arom. }), 2950,2854\left(\mathrm{CH}_{2}\right), 1697 \mathrm{br} \text {, } \\
1619(3 \mathrm{CO}), 1594,1522(\mathrm{NH}, \mathrm{C}=\mathrm{C}) .\end{array}$ \\
\hline $10 b$ & $\mathrm{CH}_{3}$ & $\begin{array}{c}\mathrm{C}_{23} \mathrm{H}_{19} \mathrm{~N}_{3} \mathrm{O}_{4} \\
401.42\end{array}$ & $\begin{array}{c}220-1 \\
41\end{array}$ & $\begin{array}{l}\text { C } 68.82 \\
\mathrm{H} \quad 4.77 \\
\mathrm{~N} 10.47\end{array}$ & $\begin{array}{l}\text { C } 68.53 \\
\mathrm{H} \quad 4.51 \\
\mathrm{~N} 10.44\end{array}$ & $\begin{array}{l}3252,3193,3133(3 \mathrm{NH}), 3082(\mathrm{CH} \\
\text { arom }), 2928,2857\left(\mathrm{CH}_{3}, \mathrm{CH}_{2}\right), 1738, \\
1689,1620(3 \mathrm{CO}), 1594,1565,1520 \\
(\mathrm{NH}, \mathrm{C}=\mathrm{C}) .\end{array}$ \\
\hline $10 \mathrm{c}$ & $\mathrm{Br}$ & $\begin{array}{c}\mathrm{C}_{22} \mathrm{H}_{16} \mathrm{BrN}_{3} \mathrm{O}_{4} \\
466.29\end{array}$ & $\begin{array}{c}141-3 \\
45\end{array}$ & $\begin{array}{lr}\text { C } & 56.67 \\
\mathrm{H} & 3.46 \\
\mathrm{~N} & 9.01 \\
\end{array}$ & $\begin{array}{lr}\mathrm{C} & 56.75 \\
\mathrm{H} & 3.64 \\
\mathrm{~N} & 9.25 \\
\end{array}$ & $\begin{array}{l}3192 \mathrm{br}(3 \mathrm{NH}), 2926\left(\mathrm{CH}_{2}\right), \\
1685 \mathrm{br}, 1621(3 \mathrm{CO}), 1595,1522 \\
(\mathrm{NH}, \mathrm{C}=\mathrm{C}) .\end{array}$ \\
\hline 10d & $\mathrm{OCH}_{3}$ & $\begin{array}{c}\mathrm{C}_{23} \mathrm{H}_{19} \mathrm{~N}_{3} \mathrm{O}_{5} \\
417.42\end{array}$ & $\begin{array}{c}198-9 \\
43\end{array}$ & $\begin{array}{l}\text { C } 66.18 \\
\mathrm{H} \quad 4.59 \\
\mathrm{~N} 10.07\end{array}$ & $\begin{array}{l}\text { C } 66.40 \\
\mathrm{H} \quad 4.63 \\
\mathrm{~N} 10.42\end{array}$ & $\begin{array}{l}3251,3193,3133(3 \mathrm{NH}), 3081 \\
(\mathrm{CH} \text { arom. }), 2963,2839\left(\mathrm{CH}_{3}, \mathrm{CH}_{2}\right), \\
1737,1690,1621(3 \mathrm{CO}), 1594, \\
1565,1513(\mathrm{NH}, \mathrm{C}=\mathrm{C}) .\end{array}$ \\
\hline $10 \mathrm{e}$ & $\mathrm{Cl}$ & $\begin{array}{c}\mathrm{C}_{22} \mathrm{H}_{16} \mathrm{ClN}_{3} \mathrm{O}_{4} \\
421.84\end{array}$ & $\begin{array}{c}165-7 \\
55\end{array}$ & $\begin{array}{lr}\mathrm{C} & 62.64 \\
\mathrm{H} & 3.82 \\
\mathrm{~N} & 9.96\end{array}$ & $\begin{array}{l}\mathrm{C} 62.84 \\
\mathrm{H} \quad 4.09 \\
\mathrm{~N} 10.15\end{array}$ & $\begin{array}{l}3251,3192,3132(3 \mathrm{NH}), 3082(\mathrm{CH} \\
\text { arom }), 2931,2854\left(\mathrm{CH}_{2}\right), 1737,1689 \\
, 1619(3 \mathrm{CO}), 1594,1565,1523(\mathrm{NH}, \\
\mathrm{C}=\mathrm{C}) .\end{array}$ \\
\hline $10 f$ & $\mathrm{COOH}$ & $\begin{array}{c}\mathrm{C}_{23} \mathrm{H}_{17} \mathrm{~N}_{3} \mathrm{O}_{6} \\
431.41\end{array}$ & $\begin{array}{c}265-7 \\
74\end{array}$ & $\begin{array}{lr}\mathrm{C} & 64.04 \\
\mathrm{H} & 3.97 \\
\mathrm{~N} & 9.74\end{array}$ & $\begin{array}{lr}\mathrm{C} & 64.23 \\
\mathrm{H} & 4.35 \\
\mathrm{~N} & 9.92\end{array}$ & $\begin{array}{l}3422,3226 \mathrm{br}(3 \mathrm{NH}), 2924\left(\mathrm{CH}_{2}\right), \\
2600 \mathrm{br}(\mathrm{OH} \text { acid }), 1687 \mathrm{br}, 1622 \\
(4 \mathrm{CO}), 1595,1522(\mathrm{NH}, \mathrm{C}=\mathrm{C})\end{array}$ \\
\hline $10 \mathrm{~g}$ & $\mathrm{NO}_{2}$ & $\begin{array}{c}\mathrm{C}_{22} \mathrm{H}_{16} \mathrm{~N}_{4} \mathrm{O}_{6} \\
432.39\end{array}$ & $\begin{array}{c}231-3 \\
56\end{array}$ & 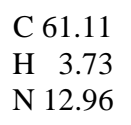 & $\begin{array}{l}\text { C } 61.62 \\
\text { H } 3.52 \\
N 12.66\end{array}$ & $\begin{array}{l}3228 \mathrm{br}(3 \mathrm{NH}), 2925\left(\mathrm{CH}_{2}\right), \\
1689 \mathrm{br}, 1621(3 \mathrm{CO}), 1593,1521 \\
(\mathrm{NH}, \mathrm{C}=\mathrm{C})\end{array}$ \\
\hline $10 \mathrm{~h}$ & $\mathrm{SO}_{2} \mathrm{NH}_{2}$ & $\begin{array}{c}\mathrm{C}_{22} \mathrm{H}_{18} \mathrm{~N}_{4} \mathrm{O}_{6} \mathrm{~S} \\
466.48\end{array}$ & $\begin{array}{c}285-7 \\
61\end{array}$ & $\begin{array}{l}\text { C } 56.65 \\
H \quad 3.89 \\
N \quad 12.01\end{array}$ & $\begin{array}{l}\text { C } 56.86 \\
\text { H } 3.92 \\
\text { N } 11.90\end{array}$ & $\begin{array}{l}3351,3288,3220 \mathrm{br}\left(\mathrm{NH}_{2}, 3 \mathrm{NH}\right), \\
3076(\mathrm{CH} \text { arom. }), 1683 \mathrm{br}, 1621 \\
(3 \mathrm{CO}), 1596,1573,1522(\mathrm{NH}, \\
\mathrm{C}=\mathrm{C}), 1324,1147\left(\mathrm{SO}_{2}\right) .\end{array}$ \\
\hline
\end{tabular}

Phenylhydrazinocarbonylmethyl 9chloroacridine-4-carboxylate 11 (Scheme 3)

A suspension of $10 \mathrm{a}(1 \mathrm{~g}, 2.58$ mmol) in thionyl chloride $(5 \mathrm{ml})$ containing 2 drops dry DMF was heated gently under reflux until a homogenous solution was obtained, then for further $30 \mathrm{~min}$. The solution was evaporated to dryness under vacuum on a water bath to remove excess thionyl chloride. The residue was azeotroped three times with dry benzene $(5 \mathrm{ml}$ each) where the last traces of thionyl chloride were removed. The residue was used directly for preparation of 12a-k. 


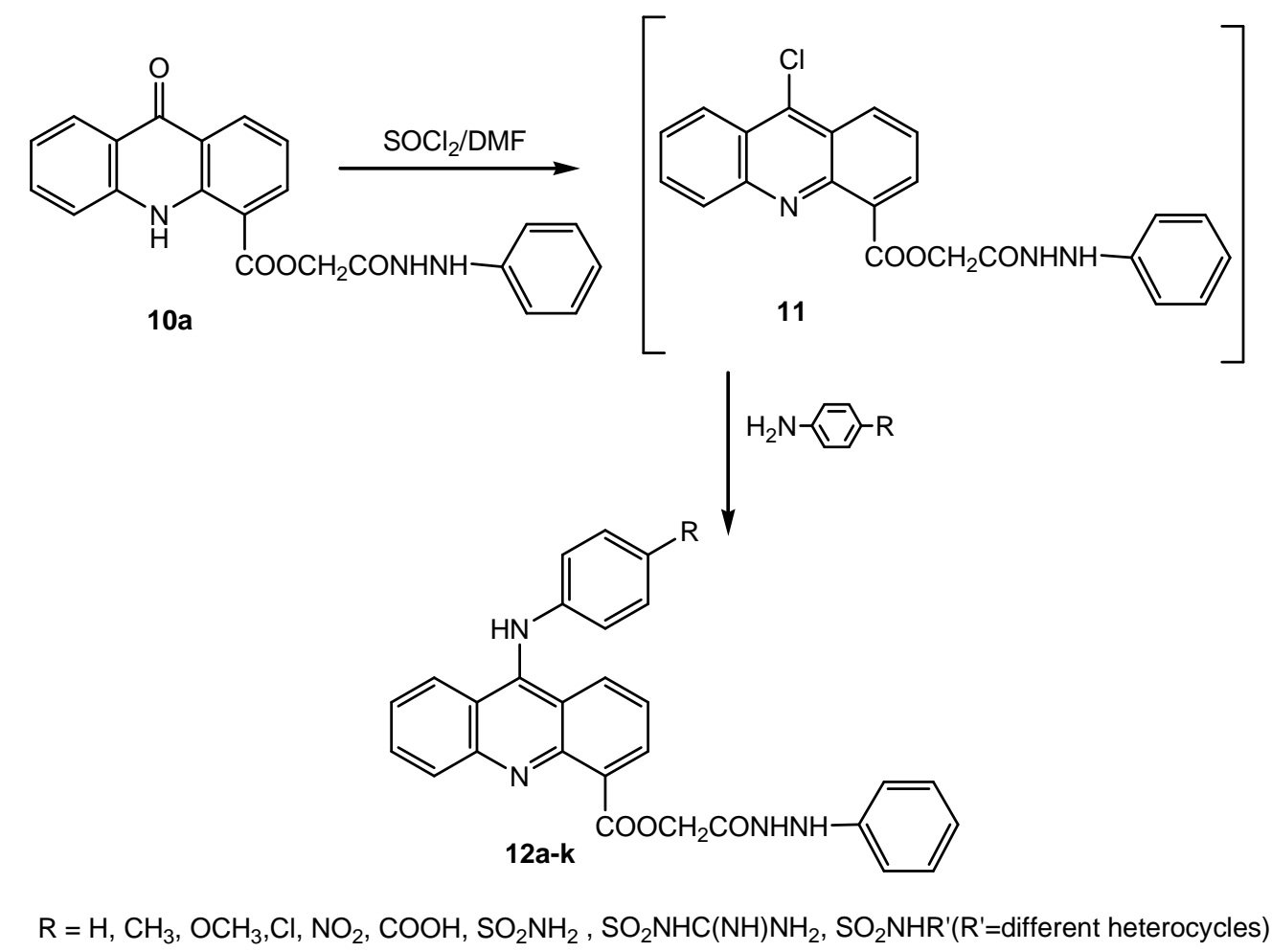

Scheme 3

Phenylhydrazinocarbonylmethyl 9(4-substituted phenyl)aminoacridine-4-carboxylate 12a-k (Scheme 3, Table 5)

A suspension of the freshly prepared $\mathbf{1 1}$ in dry DMF $(10 \mathrm{ml})$ was added to a stirred solution of equimolar amount of substituted amines $(2.58 \mathrm{mmol})$ in dry DMF (10 $\mathrm{ml}$ ) acidified with few drops of dilute $\mathrm{HCl}$ and stirring was continued for 30 $\mathrm{min}$ at room temperature. The resulting solution diluted with icewater and neutralized by dropwise addition of dilute $\mathrm{NH}_{4} \mathrm{OH}$ solution. The precipitate was filtered, washed with water, dried and crystallized from DMF/water.

12a ${ }^{1} \mathrm{H}$ NMR: $\delta=5.14(\mathrm{~s}, 2 \mathrm{H}$, $\mathrm{CH}_{2}$ ), 7.09-8.62 (m, $18 \mathrm{H}$, arom. $\underline{\mathrm{H}}$,

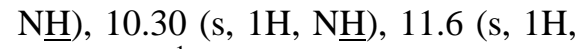
NH). 12k ${ }^{1} \mathrm{H}$ NMR: $\delta=2.28(\mathrm{~s}, 3 \mathrm{H}$, $\left.\mathrm{C}_{3}\right), 5.14$ (s, 2H, $\mathrm{C}_{2}$ ), 6.04-8.62 $(\mathrm{m}, 17 \mathrm{H}$, arom. $\underline{\mathrm{H}}), 10.38(\mathrm{~s}, 1 \mathrm{H}$, $\mathrm{NH}), 10.87$ (s, 2H, 2Nㅂ), 11.63 (s, 1H, N $\underline{H})$. 12a MS: $\mathrm{m} / \mathrm{z}(\%) \mathrm{M}^{+}, 462$ (0.51) and 256, (100). 12b MS: m/z (\%) $\mathrm{M}^{+}, 476$ (14.2) and 221, (100). 
Table 5: Physical, analytical and IR spectral data for compounds 12a-k.

\begin{tabular}{|c|c|c|c|c|c|c|}
\hline \multirow{2}{*}{$\begin{array}{l}\text { Cpd. } \\
\text { No. }\end{array}$} & \multirow{2}{*}{$\mathrm{R}$} & \multirow{2}{*}{$\begin{array}{l}\text { Mol. Formula } \\
\text { Mol. Wt. }\end{array}$} & \multirow{2}{*}{$\begin{array}{l}\text { m.p } \\
\text { Yield } \\
\%\end{array}$} & \multicolumn{2}{|c|}{ Micro analysis } & \multirow{2}{*}{ IR $\mathrm{cm}^{-1}$} \\
\hline & & & & Calc. & Found & \\
\hline $12 \mathbf{a}$ & $\mathrm{H}$ & $\begin{array}{c}\mathrm{C}_{28} \mathrm{H}_{22} \mathrm{~N}_{4} \mathrm{O}_{3} \\
462.51\end{array}$ & $\begin{array}{c}231-3 \\
79\end{array}$ & $\begin{array}{l}\text { C } 72.71 \\
\text { H } 4.79 \\
\text { N } 12.11 \\
\end{array}$ & $\begin{array}{l}\mathrm{C} 72.96 \\
\mathrm{H} \quad 4.62 \\
\mathrm{~N} 12.43\end{array}$ & $\begin{array}{l}3318(3 \mathrm{NH}), 3051(\mathrm{CH} \text { arom. }), 2927 \\
\left(\mathrm{CH}_{2}\right), 1645 \mathrm{br}(2 \mathrm{CO}), 1618,1593, \\
1554,1522(\mathrm{NH}, \mathrm{C}=\mathrm{C}, \mathrm{C}=\mathrm{N})\end{array}$ \\
\hline $12 b$ & $\mathrm{CH}_{3}$ & $\begin{array}{c}\mathrm{C}_{29} \mathrm{H}_{24} \mathrm{~N}_{4} \mathrm{O}_{3} \\
476.54\end{array}$ & $\begin{array}{c}218-20 \\
42\end{array}$ & $\begin{array}{l}\text { C } 73.09 \\
\mathrm{H} \\
\mathrm{N} 11.08 \\
\end{array}$ & $\begin{array}{lr}\text { C } 73.11 \\
\text { H } 5.20 \\
\text { N } 11.73\end{array}$ & $\begin{array}{l}3310,3257,3123(3 \mathrm{NH}), 3052(\mathrm{CH} \\
\text { arom. }), 2917,2860\left(\mathrm{CH}_{3}, \mathrm{CH}_{2}\right), 1692, \\
1668(2 \mathrm{CO}), 1620,1594,1542,1518 \\
(\mathrm{NH}, \mathrm{C}=\mathrm{C}, \mathrm{C}=\mathrm{N}) .\end{array}$ \\
\hline $12 \mathrm{c}$ & $\mathrm{OCH}_{3}$ & $\begin{array}{c}\mathrm{C}_{29} \mathrm{H}_{24} \mathrm{~N}_{4} \mathrm{O}_{4} \\
492.54\end{array}$ & $\begin{array}{c}197-9 \\
54\end{array}$ & $\begin{array}{l}\text { C } 70.72 \\
\mathrm{H} \quad 4.91 \\
\mathrm{~N} 11.38\end{array}$ & $\begin{array}{l}\mathrm{C} 70.22 \\
\mathrm{H} \quad 4.54 \\
\mathrm{~N} 11.50\end{array}$ & $\begin{array}{l}3308,3258,3123(3 \mathrm{NH}), 3051(\mathrm{CH} \\
\text { arom. }), 2953,2916\left(\mathrm{CH}_{2}, \mathrm{CH}_{2}\right), 1693, \\
1668(2 \mathrm{CO}), 1619,1594,1541,1518 \\
(\mathrm{NH}, \mathrm{C}=\mathrm{C}, \mathrm{C}=\mathrm{N}) .\end{array}$ \\
\hline $12 d$ & $\mathrm{Cl}$ & $\begin{array}{c}\mathrm{C}_{28} \mathrm{H}_{21} \mathrm{ClN}_{4} \mathrm{O}_{3} \\
496.95\end{array}$ & $\begin{array}{c}161-3 \\
57\end{array}$ & $\begin{array}{l}\mathrm{C} 67.67 \\
\mathrm{H} \quad 4.26 \\
\mathrm{~N} 11.27 \\
\end{array}$ & 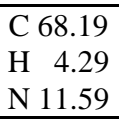 & $\begin{array}{l}3420 \mathrm{br}, 3142(3 \mathrm{NH}), 3055(\mathrm{CH} \text { arom. }), \\
2929\left(\mathrm{CH}_{2}\right), 1670 \mathrm{br}(2 \mathrm{CO}), 1626, \\
1586,1557,1516(\mathrm{NH}, \mathrm{C}=\mathrm{C}, \mathrm{C}=\mathrm{N}) .\end{array}$ \\
\hline $12 \mathrm{e}$ & $\mathrm{COOH}$ & $\begin{array}{c}\mathrm{C}_{29} \mathrm{H}_{22} \mathrm{~N}_{4} \mathrm{O}_{5} \\
506.52\end{array}$ & $\begin{array}{c}211-3 \\
61\end{array}$ & $\begin{array}{l}\text { C } 68.77 \\
\mathrm{H} \quad 4.38 \\
\mathrm{~N} 11.06\end{array}$ & $\begin{array}{l}\text { C } 69.00 \\
\mathrm{H} \quad 4.59 \\
\mathrm{~N} 11.42\end{array}$ & $\begin{array}{l}3255,3194,3133(3 \mathrm{NH}), 3082(\mathrm{CH} \\
\text { arom. }), 2985,2946\left(\mathrm{CH}_{2}\right), 2646 \mathrm{br}, \\
2528 \mathrm{br}(\mathrm{OHacid}), 1737,1687(3 \mathrm{CO}), \\
1618,1593,1570,1522(\mathrm{NH}, \mathrm{C}=\mathrm{C}, \mathrm{C}=\mathrm{N})\end{array}$ \\
\hline $12 f$ & $\mathrm{NO}_{2}$ & $\begin{array}{c}\mathrm{C}_{28} \mathrm{H}_{21} \mathrm{~N}_{5} \mathrm{O}_{5} \\
507.51\end{array}$ & $203-5$ & $\begin{array}{l}\text { C } 66.27 \\
\mathrm{H} 4.17 \\
\mathrm{~N} 13.8 \\
\end{array}$ & $\begin{array}{lr}\mathrm{C} & 66.45 \\
\mathrm{H} & 4.33 \\
\mathrm{~N} & 13.89 \\
\end{array}$ & $\begin{array}{l}3192 \mathrm{br}(3 \mathrm{NH}), 2926\left(\mathrm{CH}_{2}\right), 1687 \mathrm{br} \\
(2 \mathrm{CO}), 1622,1596,1568,1522(\mathrm{NH}, \\
\mathrm{C}=\mathrm{C}, \mathrm{C}=\mathrm{N}) .\end{array}$ \\
\hline $12 \mathrm{~g}$ & $\mathrm{SO}_{2} \mathrm{NH}_{2}$ & $\begin{array}{c}\mathrm{C}_{28} \mathrm{H}_{23} \mathrm{~N}_{5} \mathrm{O}_{5} \mathrm{~S} \\
541.59\end{array}$ & $\begin{array}{c}231-3 \\
62\end{array}$ & $\begin{array}{l}\text { C } 62.10 \\
\mathrm{H} \quad 4.28 \\
\mathrm{~N} 12.93\end{array}$ & $\begin{array}{l}\mathrm{C} 62.25 \\
\mathrm{H} \quad 4.30 \\
\mathrm{~N} 12.63\end{array}$ & $\begin{array}{l}3284 \mathrm{br}\left(\mathrm{NH}_{2}, 3 \mathrm{NH}\right), 3063(\mathrm{CH} \text { arom. }), \\
2926\left(\mathrm{CH}_{2}\right), 1660 \mathrm{br}(2 \mathrm{CO}), 1617,1578, \\
1520(\mathrm{NH}, \mathrm{C}=\mathrm{C}, \mathrm{C}=\mathrm{N}) 1329,1151 \\
\left(\mathrm{SO}_{2}\right) .\end{array}$ \\
\hline $12 \mathrm{~h}$ & & $\begin{array}{c}\mathrm{C}_{29} \mathrm{H}_{25} \mathrm{~N}_{7} \mathrm{O}_{5} \mathrm{~S} \\
583.63\end{array}$ & $\begin{array}{c}235-7 \\
49\end{array}$ & $\begin{array}{l}\text { C } 59.68 \\
\mathrm{H} \quad 4.32 \\
\mathrm{~N} 16.80 \\
\end{array}$ & $\begin{array}{l}\text { C } 59.38 \\
H \quad 4.73 \\
N \quad 16.67 \\
\end{array}$ & $\begin{array}{l}3424,3325,3209\left(\mathrm{NH}_{2}, 5 \mathrm{NH}\right), 2926 \\
\left(\mathrm{CH}_{2}\right), 1663 \mathrm{br}(2 \mathrm{CO}), 1618,1581,1542 \\
(\mathrm{NH}, \mathrm{C}=\mathrm{C}, \mathrm{C}=\mathrm{N}), 1315,1132\left(\mathrm{SO}_{2}\right)\end{array}$ \\
\hline $12 \mathbf{i}$ & & $\begin{array}{c}\mathrm{C}_{34} \mathrm{H}_{29} \mathrm{~N}_{7} \mathrm{O}_{5} \mathrm{~S} \\
647.72\end{array}$ & $\begin{array}{c}225-7 \\
42\end{array}$ & $\begin{array}{l}\text { C } 63.05 \\
\mathrm{H} \quad 4.51 \\
\mathrm{~N} 15.14\end{array}$ & $\begin{array}{l}\text { C } 63.01 \\
\text { H } 4.80 \\
\text { N } 15.31\end{array}$ & $\begin{array}{l}3423,3355,3259(4 \mathrm{NH}), 3037(\mathrm{CH} \\
\text { arom }), 2936,2870\left(\mathrm{CH}_{3}, \mathrm{CH}_{2}\right), 1694, \\
1651(2 \mathrm{CO}), 1622,1583,1494(\mathrm{NH}, \\
\mathrm{C}=\mathrm{C}, \mathrm{C}=\mathrm{N}), 1324,1154\left(\mathrm{SO}_{2}\right) .\end{array}$ \\
\hline $12 j$ & & $\begin{array}{c}\mathrm{C}_{32} \mathrm{H}_{25} \mathrm{~N}_{7} \mathrm{O}_{5} \mathrm{~S} \\
619.66\end{array}$ & $\begin{array}{c}241-2 \\
52\end{array}$ & $\begin{array}{l}\text { C } 62.03 \\
\mathrm{H} \quad 4.07 \\
\mathrm{~N} 15.82\end{array}$ & $\begin{array}{l}\text { C } 61.93 \\
\mathrm{H} \quad 3.82 \\
\mathrm{~N} 15.61\end{array}$ & $\begin{array}{l}3426,3365,3291,3249(4 \mathrm{NH}), 3061 \\
\left.\text { (CHarom.),2924( } \mathrm{CH}_{2}\right), 1695 \mathrm{br}(2 \mathrm{CO}) \\
\text { 1625,1596,1575,1525 (NH,C=C, } \\
\mathrm{C}=\mathrm{N}), 1342,1141\left(\mathrm{SO}_{2}\right)\end{array}$ \\
\hline $12 k$ & & $\begin{array}{c}\mathrm{C}_{32} \mathrm{H}_{26} \mathrm{~N}_{6} \mathrm{O}_{6} \mathrm{~S} \\
622.66\end{array}$ & $\begin{array}{c}184-6 \\
67\end{array}$ & $\begin{array}{l}\text { C } 61.73 \\
\mathrm{H} \quad 4.21 \\
\mathrm{~N} 13.50\end{array}$ & $\begin{array}{l}\text { C } 61.90 \\
\mathrm{H} \quad 4.40 \\
\mathrm{~N} 13.15\end{array}$ & $\begin{array}{l}\text { 3423,3219br }(4 \mathrm{NH}), 3061(\mathrm{CH} \text { arom. }), \\
2926\left(\mathrm{CH}_{3}, \mathrm{CH}_{2}\right), 1655 \text { br(2CO),1620, } \\
\text { 1594,1520(NH,C=C,C=N),1326, } \\
1157\left(\mathrm{SO}_{2}\right) .\end{array}$ \\
\hline
\end{tabular}


9-Oxo-9,10-dihydroacridine-4-carbonyloxyacetic acid 13 (Scheme 4)

Acridone-4-caboxylic acid 8 (1.14 $\mathrm{g}, 4.8 \mathrm{mmol}$ ) was dissolved in $2 \%$ $\mathrm{NaOH}$ solution $(5 \mathrm{ml}$ ) followed by addition of ethanol $(40 \mathrm{ml})$, the resulting solution was evaporated to dryness. The yellow residue of sodium 9-oxo-9,10-dihydroacridine4-carboxylate 9 obtained was dried then dissolved in dry DMF $(20 \mathrm{ml})$ and treated with equimolar amount of mono chloroacetic acid $(0.45 \mathrm{~g}, 4.8$ $\mathrm{mmol})$ in dry DMF $(10 \mathrm{ml})$. The reaction mixture was refluxed for 3 hrs, where $\mathrm{NaCl}$ separated out, then filtered and the filtrate was poured onto ice-water $(150 \mathrm{ml})$. The yellow precipitate was collected, washed with water and dried to give $(0.65 \mathrm{~g}$, $91 \%)$. The solid was crystallized from DMF/water. m.p. 315-7 . IR $(\mathrm{KBr})$ : 3222br $(\mathrm{NH}), 2926\left(\mathrm{CH}_{2}\right)$, 2600 br (OH acid), 1722, 1688, 1622 (3CO), 1594, $1522 \mathrm{~cm}^{-1}(\mathrm{NH}, \mathrm{C}=\mathrm{C})$. ${ }^{1} \mathrm{H}$ NMR: $\delta=4.93\left(\mathrm{~s}, 2 \mathrm{H}, \mathrm{C}_{2}\right), 7.19$ $8.42(\mathrm{~m}, 8 \mathrm{H}$, arom. $\mathrm{H}, \mathrm{NH}), 11.8(\mathrm{~s}$, $1 \mathrm{H}, \mathrm{COOH}) . \mathrm{MS}: \mathrm{m} / \mathrm{z}(\%) \mathrm{M}^{+}, 297$, (3.9) and 221, (100). Calculated $\mathrm{C}_{16} \mathrm{H}_{11} \mathrm{NO}_{5}$ (297.27), calculated $\mathrm{C}$ $64.65, \mathrm{H} 3.73, \mathrm{~N}$ 4.71. Found $\mathrm{C}$ $64.81, \mathrm{H} 3.91, \mathrm{~N} 4.42$.<smiles>O=C(O)c1cccc2c(=O)c3ccccc3[nH]c12</smiles>

8<smiles>[R]c1ccc(Nc2c3ccccc3nc3c(C(=O)OCc4ccccc4)cccc23)cc1</smiles>

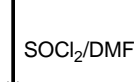<smiles>O=C(OCl)c1cccc2c(Cl)c3ccccc3nc12</smiles>

$\mid \begin{aligned} & \mathrm{H}_{2} \mathrm{~N}-\langle-1 \\ & \mathrm{TEA}\end{aligned}$<smiles>[R]OC(=O)c1cccc2c(Nc3ccc(-[14c]4ccccc4)cc3)c3ccccc3nc12</smiles><smiles>O=C(OCc1ccccc1)c1cccc2c(Cl)c3ccccc3nc12</smiles>

$\mathrm{R}=\mathrm{H}, \mathrm{CH}_{3}, \mathrm{OCH}_{3}, \mathrm{Cl}, \mathrm{NO}_{2}, \mathrm{COOH}, \mathrm{SO}_{2} \mathrm{NH}_{2}, \mathrm{SO}_{2} \mathrm{NHC}\left(\mathrm{NH}^{2} \mathrm{NH}_{2}, \mathrm{SO}_{2} \mathrm{NHR}^{\prime}\right.$ ( $\mathrm{R}^{\prime}=$ different heterocycles)

Scheme 4 
2-Chloro-2-oxoethyl 9-chloroacridine-4-carboxylate 14 (Scheme 4)

A suspension of $\mathbf{1 3}(1 \mathrm{~g}, 3.36$ mmol) in thionyl chloride $(5 \mathrm{ml})$ containing drops of dry DMF was heated gently under reflux until a homogenous solution was obtained, then for further one hr. The solution was evaporated to dryness to remove excess thionyl chloride. The residue was azeotroped three times with dry benzene $(5 \mathrm{ml}$ each) where the last traces of thionyl chloride were removed. The residue was used directly for preparation of $\mathbf{1 5 a}-\mathbf{k}$ and 17a-j.

4-Substituted phenylcarbamoylmethyl 9-(4-substituted phenyl) aminoacridine-4-carboxylate 15a-k (Scheme 4, Table 6)

A freshly prepared solution of $\mathbf{1 4}$ (3.36 mmol) dissolved in dry DMF $(10 \mathrm{ml})$ was added to stirred solution of substituted amines $(6.72 \mathrm{mmol})$ dissolved in dry DMF $(10 \mathrm{ml})$ and stirring was continued for $30 \mathrm{~min}$ at room temperature. The reaction mixture was diluted with ice-water and neutralized by dropwise addition of dilute $\mathrm{NH}_{4} \mathrm{OH}$ solution till complete precipitation. The precipitate was filtered, washed with water, dried and crystallized from DMF/water.

15a ${ }^{1} \mathrm{H}$ NMR: $\delta=4.95(\mathrm{~s}, 2 \mathrm{H}$, $\left.\mathrm{CH}_{2}\right), 7.28-8.59$ (m, $17 \mathrm{H}$, arom. $\left.\underline{\mathrm{H}}\right)$,

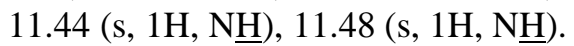
15d ${ }^{1} \mathrm{H}$ NMR: $\delta=5.17(\mathrm{~s}, 2 \mathrm{H}$,
$\mathrm{CH}_{2}$ ), 7.34-8.58 (m, $16 \mathrm{H}$, arom. $\underline{\mathrm{H}}$, $\mathrm{NH}), 11.43(\mathrm{~s}, 1 \mathrm{H}, \mathrm{NH})$. 15a MS: $\mathrm{m} / \mathrm{z}(\%) \mathrm{M}^{+}, 447,(17.24)$ and 295 , (100). 15b MS: m/z (\%) $\mathrm{M}^{+}, 475$, (51.44) and 221, (100).

\section{Phenylcarbamoylmethyl 9-(4-} substituted phenyl)aminoacridine4- carboxylate 17a-j (Scheme 4, Table 7)

To the freshly prepared solution of $14(2.17 \mathrm{mmol})$ dissolved in dry DMF $(10 \mathrm{ml})$, a solution of aniline $(0.2 \mathrm{~g}$, $2.17 \mathrm{mmol})$ in dry DMF (10 ml) buffered with few drops of triethylamine was added and stirred for $10 \mathrm{~min}$ at room temperature. The resulting solution was then added to a solution of substituted amine (2.17 mmol) dissolved in DMF $(10 \mathrm{ml})$ acidified with drops of dilute $\mathrm{HCl}$ and stirring was continued for further 30 min. The resulting solution was diluted with ice-water and neutralized by dropwise addition of dilute $\mathrm{NH}_{4} \mathrm{OH}$ solution. The precipitate was filtered, washed with water, dried and crystallized from DMF/water.

17a ${ }^{1} \mathrm{H}$ NMR: $\delta=2.12(\mathrm{~s}, 3 \mathrm{H}$, $\left.\mathrm{CH}_{3}\right), 4.74$ (s, $\left.2 \mathrm{H}, \mathrm{C}_{2}\right), 6.46-8.60$ $(\mathrm{m}, 16 \mathrm{H}$, arom. $\mathrm{H}), 10.39(\mathrm{~s}, 1 \mathrm{H}$, $\mathrm{NH}), 11.64(\mathrm{~s}, \underline{1 \mathrm{H}}, \mathrm{NH}) . \quad \mathbf{1 7 f}{ }^{1} \mathrm{H}$ NMR: $\delta=4.99\left(\mathrm{~s}, 2 \mathrm{H}, \mathrm{C}_{2}\right), 7.29$ $8.53\left(\mathrm{~m}, 18 \mathrm{H}\right.$, arom. $\left.\underline{\mathrm{H}}, \mathrm{NH}_{2}\right), 10.95$

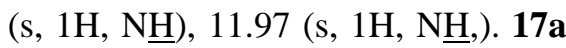
MS: $\mathrm{m} / \mathrm{z}(\%) \mathrm{M}^{+}, 461,(0.87)$ and 221, (100). 17b MS: $\mathrm{m} / \mathrm{z}(\%) \mathrm{M}^{+}$, 477, (4.19) and 221, (100). 
Table 6: Physical, analytical and IR spectral data for compounds 15a-k.

\begin{tabular}{|c|c|c|c|c|c|c|}
\hline \multirow{2}{*}{$\begin{array}{l}\text { Cpd. } \\
\text { No. }\end{array}$} & \multirow{2}{*}{$\mathrm{R}$} & \multirow{2}{*}{$\begin{array}{l}\text { Mol. Formula } \\
\text { Mol. Wt. }\end{array}$} & \multirow{2}{*}{$\begin{array}{l}\text { m.p } \\
\text { Yield } \\
\%\end{array}$} & \multicolumn{2}{|c|}{ Micro analysis } & \multirow{2}{*}{ IR $\mathrm{cm}^{-1}$} \\
\hline & & & & Calc. & Found & \\
\hline $15 \mathbf{a}$ & $\mathrm{H}$ & $\begin{array}{c}\mathrm{C}_{28} \mathrm{H}_{21} \mathrm{~N}_{3} \mathrm{O}_{3} \\
447.5\end{array}$ & $\begin{array}{c}240-2 \\
81\end{array}$ & $\begin{array}{l}\text { C } 75.15 \\
\text { H } 4.73 \\
\text { N } 9.39\end{array}$ & 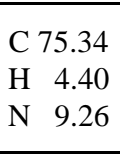 & $\begin{array}{l}3278 \text { br }(2 \mathrm{NH}), 3057(\mathrm{CH} \text { arom. }), \\
2965,2924\left(\mathrm{CH}_{2}\right), 1730,1691 \\
(2 \mathrm{CO}), 1653,1600,1555,1519(\mathrm{NH}, \\
\mathrm{C}=\mathrm{C}, \mathrm{C}=\mathrm{N})\end{array}$ \\
\hline $15 b$ & $\mathrm{CH}_{3}$ & $\begin{array}{c}\mathrm{C}_{30} \mathrm{H}_{25} \mathrm{~N}_{3} \mathrm{O}_{3} \\
475.55\end{array}$ & $\begin{array}{c}243-5 \\
68\end{array}$ & $\begin{array}{lr}\mathrm{C} & 75.77 \\
\mathrm{H} & 5.30 \\
\mathrm{~N} & 8.84\end{array}$ & $\begin{array}{lr}\mathrm{C} & 76.15 \\
\mathrm{H} & 4.99 \\
\mathrm{~N} & 8.81\end{array}$ & $\begin{array}{l}3423,3303(2 \mathrm{NH}), 3032(\mathrm{CH} \\
\text { arom. }), 2921,2857\left(\mathrm{CH}_{3}, \mathrm{CH}_{2}\right), \\
1742,1662(2 \mathrm{CO}), 1619,1586, \\
1559,1514(\mathrm{NH}, \mathrm{C}=\mathrm{C}, \mathrm{C}=\mathrm{N}) .\end{array}$ \\
\hline $15 c$ & $\mathrm{OCH}_{3}$ & $\begin{array}{c}\mathrm{C}_{30} \mathrm{H}_{25} \mathrm{~N}_{3} \mathrm{O}_{5} \\
507.55\end{array}$ & $\begin{array}{c}208-9 \\
71\end{array}$ & $\begin{array}{lr}\mathrm{C} & 70.99 \\
\mathrm{H} & 4.96 \\
\mathrm{~N} & 8.28\end{array}$ & $\begin{array}{lr}\text { C } & 70.66 \\
\mathrm{H} & 5.21 \\
\mathrm{~N} & 8.42\end{array}$ & $\begin{array}{l}3249(2 \mathrm{NH}), 3061(\mathrm{CH} \text { arom. }), \\
2930,2836\left(\mathrm{CH}_{3}, \mathrm{CH}_{2}\right), 1742, \\
1667(2 \mathrm{CO}), 1619,1593,1558, \\
1510(\mathrm{NH}, \mathrm{C}=\mathrm{C}, \mathrm{C}=\mathrm{N}) .\end{array}$ \\
\hline $15 d$ & $\mathrm{Cl}$ & $\begin{array}{c}\mathrm{C}_{28} \mathrm{H}_{19} \mathrm{Cl}_{2} \mathrm{~N}_{3} \mathrm{O}_{3} \\
516.39\end{array}$ & $\begin{array}{c}181-2 \\
63\end{array}$ & $\begin{array}{lr}\mathrm{C} & 65.13 \\
\mathrm{H} & 3.71 \\
\mathrm{~N} & 8.14 \\
\end{array}$ & $\begin{array}{lr}\mathrm{C} & 64.98 \\
\mathrm{H} & 3.55 \\
\mathrm{~N} & 7.83 \\
\end{array}$ & $\begin{array}{l}\text { 3254br(2NH),3061(CH arom. }), \\
\left.\text { 2926( } \mathrm{CH}_{2}\right), 1655 \mathrm{br}(2 \mathrm{CO}), 1615, \\
1583,1557,1513(\mathrm{NH}, \mathrm{C}=\mathrm{C}, \mathrm{C}=\mathrm{N}) .\end{array}$ \\
\hline $15 e$ & $\mathrm{COOH}$ & $\begin{array}{c}\mathrm{C}_{30} \mathrm{H}_{21} \mathrm{~N}_{3} \mathrm{O}_{7} \\
535.51\end{array}$ & $\begin{array}{c}226-8 \\
79\end{array}$ & $\begin{array}{lr}\mathrm{C} & 67.29 \\
\mathrm{H} & 3.95 \\
\mathrm{~N} & 7.85 \\
\end{array}$ & $\begin{array}{lr}\mathrm{C} & 67.59 \\
\mathrm{H} & 3.94 \\
\mathrm{~N} & 7.85 \\
\end{array}$ & $\begin{array}{l}\text { 3253,3133(2NH),3081(CHarom), } \\
2670 \mathrm{br}(\mathrm{OH} \text { acid), 1736, } 1688 \\
(4 \mathrm{CO}), 1615,1523(\mathrm{NH}, \mathrm{C}=\mathrm{C}, \mathrm{C}=\mathrm{N})\end{array}$ \\
\hline $15 f$ & $\mathrm{NO}_{2}$ & $\begin{array}{c}\mathrm{C}_{28} \mathrm{H}_{19} \mathrm{~N}_{5} \mathrm{O}_{7} \\
537.49\end{array}$ & $\begin{array}{c}221-3 \\
55\end{array}$ & $\begin{array}{l}\text { C } 62.57 \\
\mathrm{H} \\
\mathrm{N} 13.56 \\
\end{array}$ & 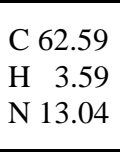 & $\begin{array}{l}3252,3194(2 \mathrm{NH}), 3082(\mathrm{CHarom}), \\
2930\left(\mathrm{CH}_{2}\right), 1737,1689(2 \mathrm{CO}), \\
1621,1596,1567,1524(\mathrm{NH}, \mathrm{C}=\mathrm{C}, \\
\mathrm{C}=\mathrm{N}) .\end{array}$ \\
\hline $15 \mathrm{~g}$ & $\mathrm{SO}_{2} \mathrm{NH}_{2}$ & $\begin{array}{c}\mathrm{C}_{28} \mathrm{H}_{23} \mathrm{~N}_{5} \mathrm{O}_{7} \mathrm{~S}_{2} \\
605.65\end{array}$ & $\begin{array}{c}245-7 \\
76\end{array}$ & $\begin{array}{l}\text { C } 55.53 \\
\mathrm{H} \quad 3.83 \\
\mathrm{~N} 11.56\end{array}$ & $\begin{array}{l}\text { C } 55.40 \\
\text { H } 3.70 \\
\text { N } 11.46\end{array}$ & $\begin{array}{l}3255 \mathrm{br}\left(2 \mathrm{NH}_{2}, 2 \mathrm{NH}\right), 3055(\mathrm{CH} \\
\text { arom }), 2928\left(\mathrm{CH}_{2}\right), 1627 \mathrm{br}(2 \mathrm{CO}), \\
1587,1552,1518(\mathrm{NH}, \mathrm{C}=\mathrm{C}, \mathrm{C}=\mathrm{N}), \\
1333,1155\left(\mathrm{SO}_{2}\right) .\end{array}$ \\
\hline $15 h$ & & $\begin{array}{c}\mathrm{C}_{30} \mathrm{H}_{27} \mathrm{~N}_{9} \mathrm{O}_{7} \mathrm{~S}_{2} \\
689.73\end{array}$ & $\begin{array}{c}251-3 \\
69\end{array}$ & $\begin{array}{l}\mathrm{C} 52.24 \\
\mathrm{H} \\
\mathrm{N} \\
\mathrm{N} 18.95 \\
\end{array}$ & 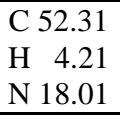 & $\begin{array}{l}3424,3197\left(2 \mathrm{NH}_{2}, 6 \mathrm{NH}\right), 1696 \mathrm{br} \\
(2 \mathrm{CO}), 1623,1591,1525(\mathrm{NH}, \\
\mathrm{C}=\mathrm{C}, \mathrm{C}=\mathrm{N}), 1271,1138\left(\mathrm{SO}_{2}\right) .\end{array}$ \\
\hline $15 \mathrm{i}$ & & $\begin{array}{c}\mathrm{C}_{40} \mathrm{H}_{35} \mathrm{~N}_{9} \mathrm{O}_{7} \mathrm{~S}_{2} \\
817.91\end{array}$ & $\begin{array}{c}220-2 \\
61\end{array}$ & $\begin{array}{l}\text { C } 58.74 \\
\mathrm{H} \quad 4.31 \\
\mathrm{~N} 15.41\end{array}$ & $\begin{array}{l}\text { C } 58.71 \\
\mathrm{H} \quad 4.20 \\
\mathrm{~N} 15.01\end{array}$ & $\begin{array}{l}3423,3355,3255,3194(4 \mathrm{NH}), \\
3075(\mathrm{CH} \text { arom. }), 2936,2870\left(\mathrm{CH}_{3} \text {, }\right. \\
\left.\mathrm{CH}_{2}\right), 1689,1651(2 \mathrm{CO}), 1620, \\
1586,1494(\mathrm{NH}, \mathrm{C}=\mathrm{C}, \mathrm{C}=\mathrm{N}), \\
1324,1153\left(\mathrm{SO}_{2}\right) .\end{array}$ \\
\hline $15 \mathbf{j}$ & & $\begin{array}{c}\mathrm{C}_{36} \mathrm{H}_{27} \mathrm{~N}_{9} \mathrm{O}_{7} \mathrm{~S}_{2} \\
761.8\end{array}$ & $\begin{array}{c}238-9 \\
59\end{array}$ & $\begin{array}{l}\text { C } 56.76 \\
\mathrm{H} \quad 3.57 \\
\mathrm{~N} 16.55\end{array}$ & $\begin{array}{l}\text { C } 57.02 \\
\mathrm{H} 4.00 \\
\mathrm{~N} 16.32\end{array}$ & $\begin{array}{l}3425,3364,3248,3134(4 \mathrm{NH}), \\
3059(\mathrm{CH} \text { arom. }), 2925\left(\mathrm{CH}_{2}\right), \\
1688,1636(2 \mathrm{CO}), 1573,1501 \\
(\mathrm{NH}, \mathrm{C}=\mathrm{C}, \mathrm{C}=\mathrm{N}), 1341,1142\left(\mathrm{SO}_{2}\right) .\end{array}$ \\
\hline $15 k$ & & $\begin{array}{c}\mathrm{C}_{36} \mathrm{H}_{29} \mathrm{~N}_{7} \mathrm{O}_{9} \mathrm{~S}_{2} \\
767.80\end{array}$ & $\begin{array}{c}215-7 \\
66\end{array}$ & $\begin{array}{lr}\mathrm{C} & 56.32 \\
\mathrm{H} & 3.81 \\
\mathrm{~N} & 12.77\end{array}$ & $\begin{array}{l}\text { C } 56.42 \\
\text { H } 3.76 \\
N \\
N\end{array}$ & $\begin{array}{l}3259 \mathrm{br}(4 \mathrm{NH}), 1691 \mathrm{br}(2 \mathrm{CO}), \\
1620,1594,1525(\mathrm{NH}, \mathrm{C}=\mathrm{C}, \mathrm{C}=\mathrm{N}), \\
1326,1163\left(\mathrm{SO}_{2}\right)\end{array}$ \\
\hline
\end{tabular}


Table 7: Physical, analytical and IR spectral data for compounds 17a-j.

\begin{tabular}{|c|c|c|c|c|c|c|}
\hline \multirow{2}{*}{$\begin{array}{l}\text { Cpd. } \\
\text { No. }\end{array}$} & \multirow{2}{*}{$\mathrm{R}$} & \multirow{2}{*}{$\begin{array}{l}\text { Mol. Formula } \\
\text { Mol. Wt. }\end{array}$} & \multirow{2}{*}{$\begin{array}{l}\text { m.p }{ }^{\circ} \\
\text { Yield } \\
\%\end{array}$} & \multicolumn{2}{|c|}{ Micro analysis } & \multirow{2}{*}{ IR $\mathrm{cm}^{-1}$} \\
\hline & & & & Calc. & Found & \\
\hline $17 \mathbf{a}$ & $\mathrm{CH}_{3}$ & $\begin{array}{c}\mathrm{C}_{29} \mathrm{H}_{23} \mathrm{~N}_{3} \mathrm{O}_{3} \\
461.52\end{array}$ & $\begin{array}{c}247-9 \\
51\end{array}$ & $\begin{array}{lr}\mathrm{C} & 75.47 \\
\mathrm{H} & 5.02 \\
\mathrm{~N} & 9.10\end{array}$ & $\begin{array}{lr}\mathrm{C} & 75.18 \\
\mathrm{H} & 4.83 \\
\mathrm{~N} & 9.08\end{array}$ & $\begin{array}{l}3423,3254(2 \mathrm{NH}), 3060 \text { (CHarom.), } \\
2923\left(\mathrm{CH}_{3}, \mathrm{CH}_{2}\right), 1742,1687(2 \mathrm{CO}), \\
1620,1593,1557,1518(\mathrm{NH}, \mathrm{C}=\mathrm{C}, \\
\mathrm{C}=\mathrm{N})\end{array}$ \\
\hline $17 b$ & $\mathrm{OCH}_{3}$ & $\begin{array}{c}\mathrm{C}_{29} \mathrm{H}_{23} \mathrm{~N}_{3} \mathrm{O}_{4} \\
477.52\end{array}$ & $\begin{array}{c}230-2 \\
54\end{array}$ & $\begin{array}{lr}\mathrm{C} & 72.94 \\
\mathrm{H} & 4.85 \\
\mathrm{~N} & 8.80\end{array}$ & $\begin{array}{lr}\mathrm{C} & 73.21 \\
\mathrm{H} & 4.81 \\
\mathrm{~N} & 8.42\end{array}$ & $\begin{array}{l}3446,3249(2 \mathrm{NH}), 3062(\mathrm{CHarom} .), \\
2930\left(\mathrm{CH}_{3}, \mathrm{CH}_{2}\right), 1741,1667(2 \mathrm{CO}), \\
1619,1593,1557,1512(\mathrm{NH}, \mathrm{C}=\mathrm{C}, \\
\mathrm{C}=\mathrm{N}) .\end{array}$ \\
\hline $17 \mathrm{c}$ & $\mathrm{Cl}$ & $\begin{array}{c}\mathrm{C}_{28} \mathrm{H}_{20} \mathrm{ClN}_{3} \mathrm{O}_{3} \\
481.94\end{array}$ & $\begin{array}{c}190-2 \\
41\end{array}$ & $\begin{array}{lr}\mathrm{C} & 69.78 \\
\mathrm{H} & 4.18 \\
\mathrm{~N} & 8.72 \\
\end{array}$ & $\begin{array}{lr}\mathrm{C} & 69.36 \\
\mathrm{H} & 4.61 \\
\mathrm{~N} & 8.72 \\
\end{array}$ & $\begin{array}{l}\text { 3396,3276(2NH),3063(CHarom.), } \\
2925\left(\mathrm{CH}_{2}\right), 1656 \mathrm{br}(2 \mathrm{CO}), 1614, \\
1583,1558,1512(\mathrm{NH}, \mathrm{C}=\mathrm{C}, \mathrm{C}=\mathrm{N}) .\end{array}$ \\
\hline $17 d$ & $\mathrm{COOH}$ & $\begin{array}{c}\mathrm{C}_{29} \mathrm{H}_{21} \mathrm{~N}_{3} \mathrm{O}_{5} \\
491.50\end{array}$ & $\begin{array}{c}251-3 \\
60\end{array}$ & $\begin{array}{lr}\mathrm{C} & 70.87 \\
\mathrm{H} & 4.31 \\
\mathrm{~N} & 8.55 \\
\end{array}$ & $\begin{array}{lr}\mathrm{C} & 70.45 \\
\mathrm{H} & 4.48 \\
\mathrm{~N} & 8.22 \\
\end{array}$ & $\begin{array}{l}\text { 3352,3252(2NH),3023(CHarom), } \\
\text { 2668br(OHacid),1735,1685(3CO), } \\
1605,1543,1521(\mathrm{NH}, \mathrm{C}=\mathrm{C}, \mathrm{C}=\mathrm{N}) .\end{array}$ \\
\hline $17 e$ & $\mathrm{NO}_{2}$ & $\begin{array}{c}\mathrm{C}_{28} \mathrm{H}_{20} \mathrm{~N}_{4} \mathrm{O}_{5} \\
492.49\end{array}$ & $\begin{array}{c}236-8 \\
45\end{array}$ & $\begin{array}{l}\mathrm{C} 68.29 \\
\mathrm{H} \quad 4.09 \\
\mathrm{~N} 11.38 \\
\end{array}$ & $\begin{array}{lr}\mathrm{C} & 68.24 \\
\mathrm{H} & 4.00 \\
\mathrm{~N} & 11.58 \\
\end{array}$ & $\begin{array}{l}\text { 3251,3194(2NH),3081(CH arom.), } \\
1736,1689(2 \mathrm{CO}), 1621,1595,1567, \\
1523(\mathrm{NH}, \mathrm{C}=\mathrm{C}, \mathrm{C}=\mathrm{N}) .\end{array}$ \\
\hline $17 f$ & $\mathrm{SO}_{2} \mathrm{I}$ & $\begin{array}{c}\mathrm{C}_{28} \mathrm{H}_{22} \mathrm{~N}_{4} \mathrm{O}_{5} \mathrm{~S} \\
526.57\end{array}$ & $\begin{array}{l}253-5 \\
79\end{array}$ & $\begin{array}{l}\text { C } 63.87 \\
\mathrm{H} \quad 4.21 \\
\mathrm{~N} 10.64\end{array}$ & 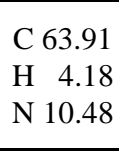 & $\begin{array}{l}3285,3186\left(\mathrm{NH}_{2}, 2 \mathrm{NH}\right), 3055(\mathrm{CH} \\
\text { arom. }), 2927\left(\mathrm{CH}_{2}\right), 1657 \mathrm{br}(2 \mathrm{CO}), \\
1612,1556,1518(\mathrm{NH}, \mathrm{C}=\mathrm{C}, \mathrm{C}=\mathrm{N}), \\
1330,1149\left(\mathrm{SO}_{2}\right) .\end{array}$ \\
\hline $17 \mathrm{~g}$ & & $\begin{array}{c}\mathrm{C}_{29} \mathrm{H}_{24} \mathrm{~N}_{6} \mathrm{O}_{5} \mathrm{~S} \\
568.61\end{array}$ & $\begin{array}{c}261-2 \\
49\end{array}$ & $\begin{array}{l}\mathrm{C} 61.26 \\
\mathrm{H} \quad 4.25 \\
\mathrm{~N} 14.78\end{array}$ & $\begin{array}{l}\text { C } 61.20 \\
\text { H } 4.50 \\
\text { N } 14.65\end{array}$ & $\begin{array}{l}3422,3337,3204\left(\mathrm{NH}_{2}, 4 \mathrm{NH}\right), 3062 \\
(\mathrm{CHarom}), 2926\left(\mathrm{CH}_{2}\right), 1742,1656 \\
(2 \mathrm{CO}), 1621,1593,1543(\mathrm{NH}, \mathrm{C}=\mathrm{C}, \\
\mathrm{C}=\mathrm{N}), 1336,1135\left(\mathrm{SO}_{2}\right) .\end{array}$ \\
\hline $17 \mathrm{~h}$ & & $\begin{array}{c}\mathrm{C}_{34} \mathrm{H}_{28} \mathrm{~N}_{6} \mathrm{O}_{5} \mathrm{~S} \\
632.7\end{array}$ & $\begin{array}{c}223-5 \\
42\end{array}$ & $\begin{array}{l}\text { C } 64.55 \\
\text { H } 4.46 \\
\text { N } 13.28\end{array}$ & $\begin{array}{l}\text { C } 64.73 \\
\text { H } 4.34 \\
\text { N } 12.97\end{array}$ & $\begin{array}{l}3423,3355,3255(3 \mathrm{NH}), 3076(\mathrm{CH} \\
\text { arom. }), 2936,2870\left(\mathrm{CH}_{3}, \mathrm{CH}_{2}\right), 1737, \\
1689(2 \mathrm{CO}), 1653,1621,1586,1494 \\
(\mathrm{NH}, \mathrm{C}=\mathrm{C}, \mathrm{C}=\mathrm{N}), 1324,1154\left(\mathrm{SO}_{2}\right) .\end{array}$ \\
\hline $17 \mathbf{i}$ & $x^{2}$ & $\begin{array}{c}\mathrm{C}_{32} \mathrm{H}_{24} \mathrm{~N}_{6} \mathrm{O}_{5} \mathrm{~S} \\
604.65\end{array}$ & $\begin{array}{c}250-2 \\
43\end{array}$ & $\begin{array}{l}\text { C } 63.57 \\
\mathrm{H} \quad 4.00 \\
\mathrm{~N} 13.90\end{array}$ & $\begin{array}{l}\text { C } 63.47 \\
\mathrm{H} \\
\mathrm{N} \\
\mathrm{N} \\
13.81\end{array}$ & $\begin{array}{l}3425,3361,3251(3 \mathrm{NH}), 3082(\mathrm{CH} \\
\text { arom. }), 1737,1690(2 \mathrm{CO}), 1621, \\
1594,1571,1527(\mathrm{NH}, \mathrm{C}=\mathrm{C}, \mathrm{C}=\mathrm{N}) \\
1326,1142\left(\mathrm{SO}_{2}\right) .\end{array}$ \\
\hline $\mathbf{1 7} \mathbf{j}$ & & $\begin{array}{c}\mathrm{C}_{32} \mathrm{H}_{25} \mathrm{~N}_{5} \mathrm{O}_{6} \mathrm{~S} \\
607.65\end{array}$ & $\begin{array}{c}216-8 \\
53\end{array}$ & $\begin{array}{l}\text { C } 63.25 \\
\text { H } 4.15 \\
\text { N } 11.53\end{array}$ & $\begin{array}{l}\text { C } 63.35 \\
\mathrm{H} \quad 4.25 \\
\mathrm{~N} 11.48\end{array}$ & $\begin{array}{l}3422 \mathrm{br}(3 \mathrm{NH}), 3061(\mathrm{CHarom} .), \\
2925\left(\mathrm{CH}_{3}, \mathrm{CH}_{2}\right), 1741,1657(2 \mathrm{CO}), \\
1619,1582,1515(\mathrm{NH}, \mathrm{C}=\mathrm{C}, \mathrm{C}=\mathrm{N}), \\
1332,1161\left(\mathrm{SO}_{2}\right) .\end{array}$ \\
\hline
\end{tabular}


Antitumor Screening

Seventeen selected compounds 10a, $10 \mathrm{~g}, 10 \mathrm{~h}, 12 \mathrm{a}, 12 \mathrm{~d}, 12 \mathrm{~g}, 12 \mathrm{~h}, 12 \mathrm{k}$, 15a, 15c, 15g, 15h, 15k, 17a, 17f, $\mathbf{1 7} \mathrm{g}$ and $\mathbf{1 7} \mathbf{j}$, were tested against the tumor cell line MCF7 (Breast carcinoma cell line) using the method of Skehan et $\mathrm{al}^{21}$ at National Cancer Institute, Cairo University.

\section{Procedure}

Cells were plated in 96-multiwell plate $\left(10^{4}\right.$ cells/well) for $24 \mathrm{hrs}$ before treatment with the compounds to allow attachment of cell to the wall of the plate. Different concentration of the compounds under test $(0,1,2.5,5$ and $10 \mathrm{~g} / \mathrm{ml}$ ) were added to the cell monolayer. Triplicate wells were prepared for each individual dose. Monolayer cells were incubated with the compounds for $48 \mathrm{hr}$ at $37^{\circ}$ and in atmosphere of $5 \% \mathrm{CO}_{2}$. After $48 \mathrm{hrs,}$ cells were fixed, washed and stained with sulforhodamine B stain. Excess stain was washed with acetic acid and attached stain was recovered with Tri EDTA buffer. Color intensity was measured in an ELISA reader. The

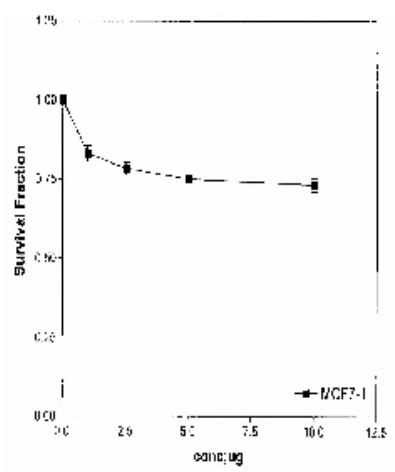

Fig. 1:Cytotoxic activity of compound 10a. relation between the surviving fraction and drug concentration was plotted to get the survival curve of each tumor cell line after the specified compound Fig. 1-17.

$\mathrm{IC}_{50}$ of the tested compounds were illustrated in Table 8.

Table 8: $\mathrm{IC}_{50}$ of tested compounds for antitumor screening.

\begin{tabular}{||c|c|c|}
\hline \multirow{2}{*}{ Fig. } & \multicolumn{2}{|c|}{ Cell line MCF7 } \\
\cline { 2 - 3 } & Compound & IC $_{50}$ \\
\hline 1 & $\mathbf{1 0 a}$ & - ve \\
\hline 2 & $\mathbf{1 0 g}$ & - ve \\
\hline 3 & $\mathbf{1 0 h}$ & - ve \\
\hline 4 & $\mathbf{1 2 a}$ & - ve \\
\hline 5 & $\mathbf{1 2 d}$ & - ve \\
\hline 6 & $\mathbf{1 2 g}$ & 9.67 \\
\hline 7 & $\mathbf{1 2 h}$ & 10 \\
\hline 8 & $\mathbf{1 2 k}$ & 4.85 \\
\hline 9 & $\mathbf{1 5 a}$ & $-\mathrm{ve}$ \\
\hline 10 & $\mathbf{1 5 c}$ & $-\mathrm{ve}$ \\
\hline 11 & $\mathbf{1 5 g}$ & 9.53 \\
\hline 12 & $\mathbf{1 5 h}$ & 7.46 \\
\hline 13 & $\mathbf{1 5 k}$ & $-\mathrm{ve}$ \\
\hline 14 & $\mathbf{1 7 a}$ & $-\mathrm{a}$ \\
\hline 15 & $\mathbf{1 7 f}$ & 4.75 \\
\hline 16 & $\mathbf{1 7 g}$ & 9.50 \\
\hline 17 & $\mathbf{1 7} \mathbf{j}$ & 7.72 \\
\hline
\end{tabular}

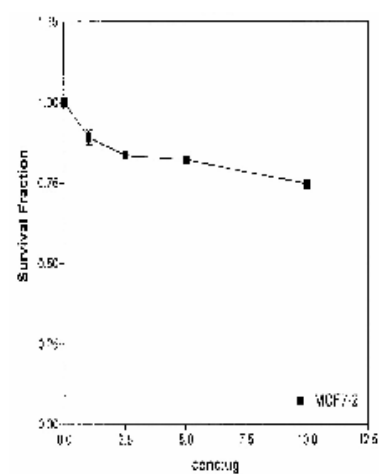

Fig. 2: Cytotoxic activity of compound $\mathbf{1 0 g}$. 


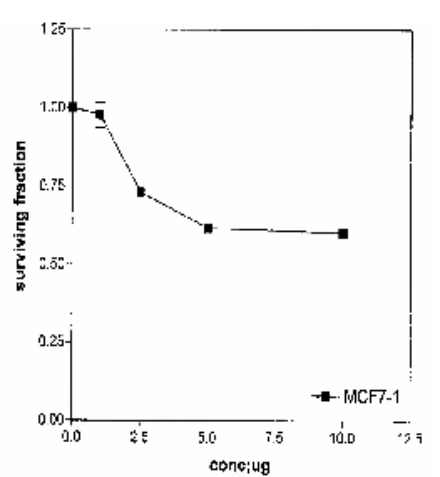

Fig. 3: Cytotoxic activity of compound $10 \mathrm{~h}$.

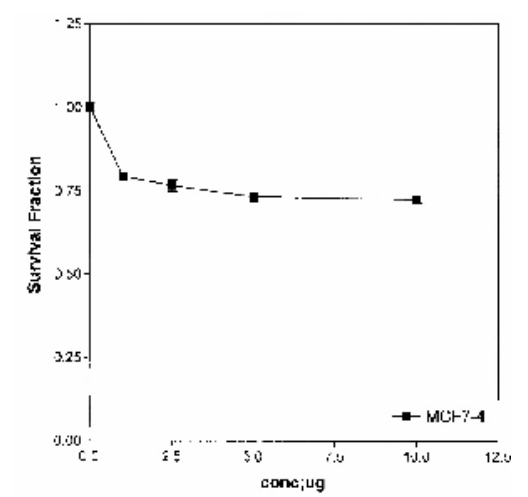

Fig. 5: Cytotoxic activity of compound $12 d$.

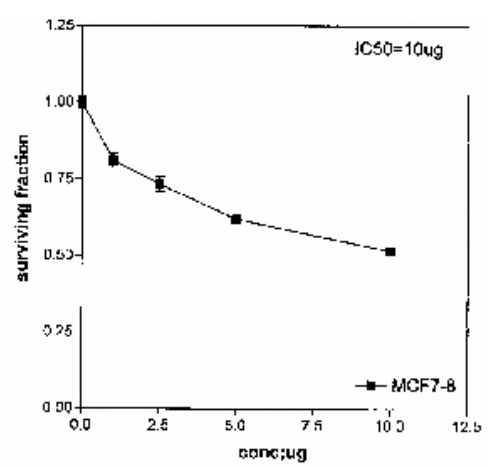

Fig. 7: Cytotoxic activity of compound $12 \mathrm{~h}$.

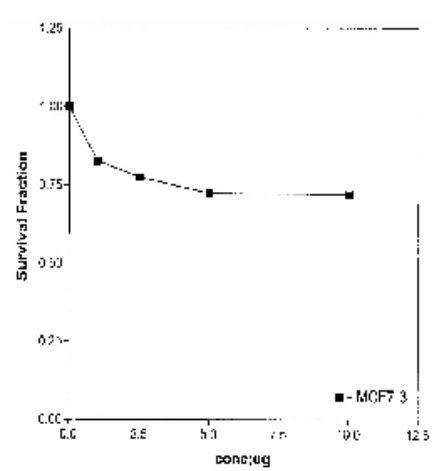

Fig. 4: Cytotoxic activity of compound $12 a$.

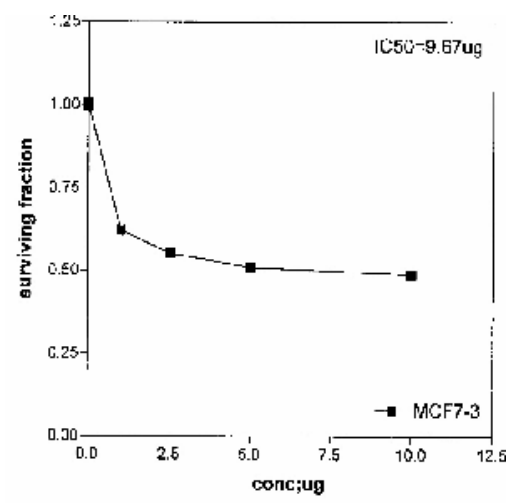

Fig. 6: Cytotoxic activity of compound $12 \mathrm{~g}$.

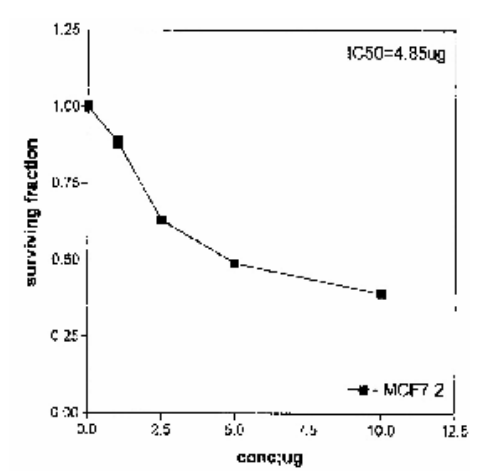

Fig. 8: Cytotoxic activity of compound 12k. 


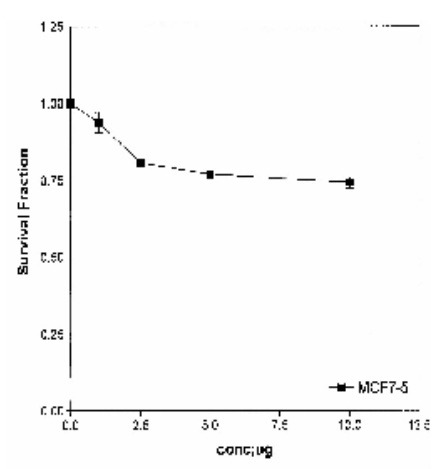

Fig. 9: Cytotoxic activity of compound $15 a$.

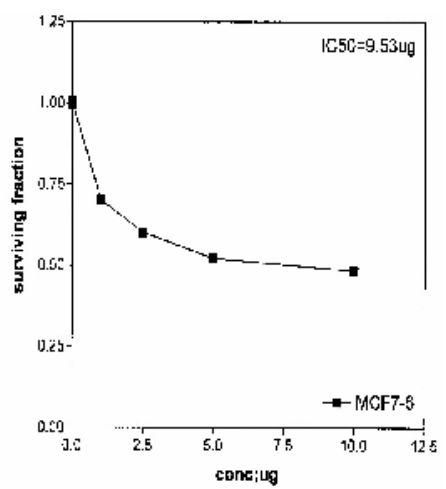

Fig. 11: Cytotoxic activity of compound $15 \mathrm{~g}$.

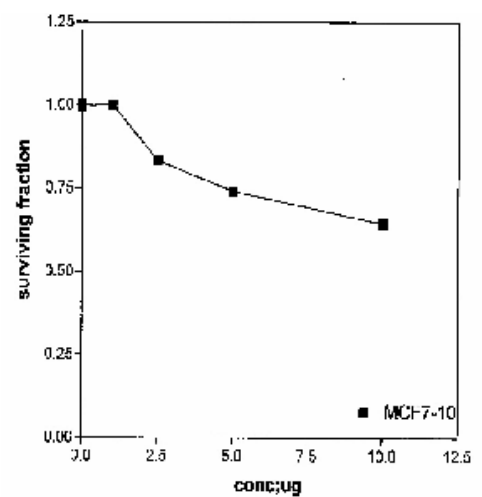

Fig. 13: Cytotoxic activity of compound 15k.

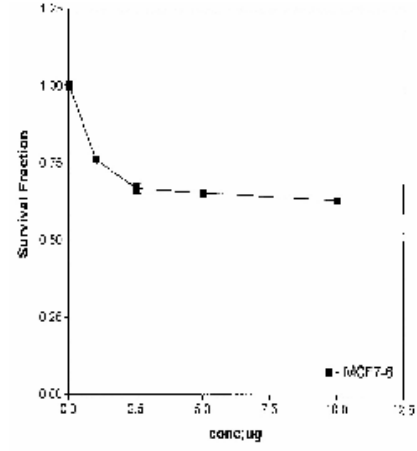

Fig. 10: Cytotoxic activity of compound $15 \mathrm{c}$.

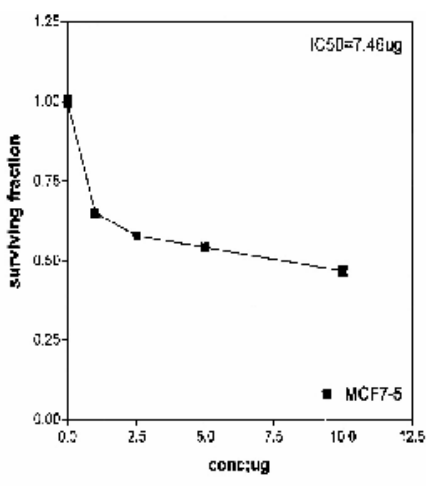

Fig. 12: Cytotoxic activity of compound $15 \mathrm{~h}$.

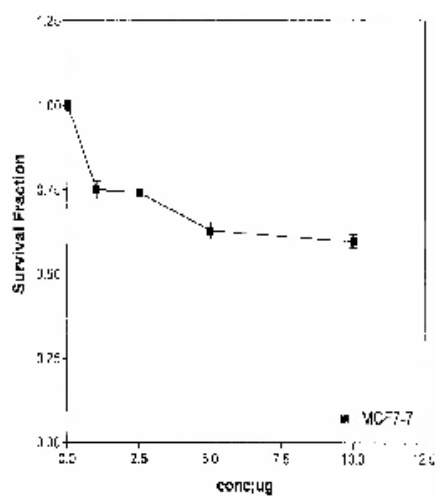

Fig. 14: Cytotoxic activity of compound 17 a. 


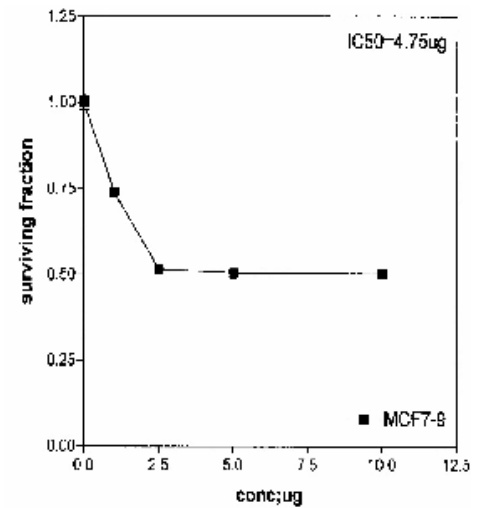

Fig. 15: Cytotoxic activity of compound $17 f$.

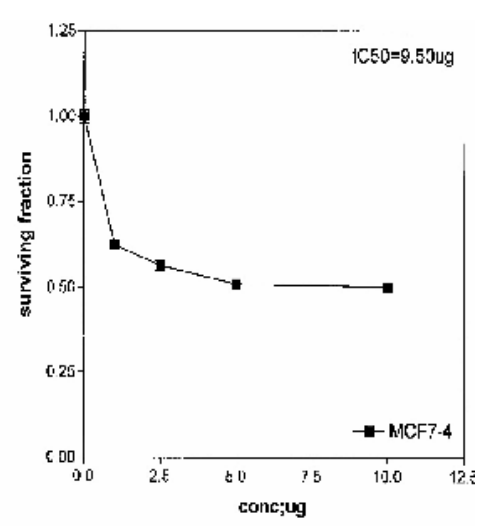

Fig. 16: Cytotoxic activity of compound $17 \mathrm{~g}$.

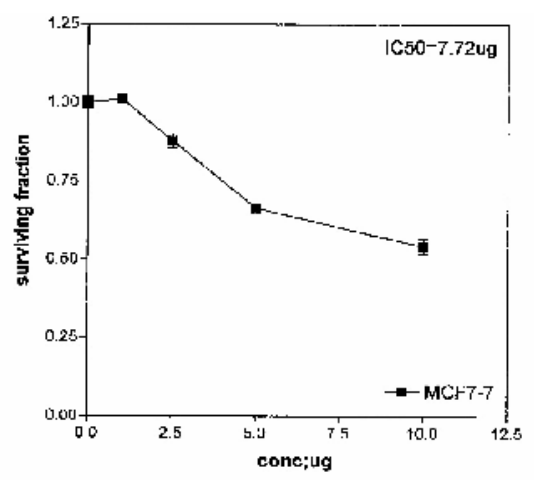

Fig. 17: Cytotoxic activity of compound $\mathbf{1 7}$ j.

\section{RESULTS AND DISCUSSION}

\section{Torsion angle}

Torsion angle comparative study results of compounds I, II and the prepared target compounds $\mathbf{1 0 h}$ revealed that a comparable torsion angle $(10,4 \mathrm{a}, 4, \mathrm{CO})$ and torsion angle (10a, 8a, 9, 9a) of compounds $\mathbf{I}$, II and $\mathbf{1 0 h}$ indicating similar alignment of the carbonyl function in all compounds.
Torsion angle comparative study results of compounds III, IV and the prepared target compounds $\mathbf{1 2 g}, \mathbf{1 5 g}$ and $\mathbf{1 7 f}$ showed that comparable torsion angle $(10,4 \mathrm{a}, 4, \mathrm{CO})$ in compounds 12g, 15g and 17f with that of compound III indicating similar alignment of carbonyl function. Also torsion angle $(9,11$, 12, 13) and torsion angle (9a, 9, 11, 12) of compounds $\mathbf{1 2 g}, \mathbf{1 5 g}$ and $\mathbf{1 7 f}$ are comparable with that of 
compound IV indicating similar alignment of 9-anilino moiety.

\section{Chemistry}

The synthetic pathway utilized to obtain the target compounds 10a-h, 12a-k, 15a-k and 17a-j is illustrated in Schemes 1-4.

The substituted phenylhydrazines 3b-h were prepared via diazotization of different aromatic amines or sulfonamides $\mathbf{1 b}-\mathbf{h}$ with sodium nitrite and hydrochloric acid followed by reduction with sodium sulfite in acidic medium to give substituted phenylhydrazine hydrochloride salts 2b-h. The free bases $\mathbf{3 b}$-h were obtained using sodium acetate ${ }^{14-17}$.

Chloroacetic acid, 2-(substituted phenyl)hydrazide 4a-h were obtained by dropwise addition of chloroacetyl chloride into a solution of the corresponding phenylhydrazine 3a-h in dry benzene at room temperature. IR spectra revealed aliphatic $\mathrm{CH}_{2}$ stretching at about 2964, $2840 \mathrm{~cm}^{-1}$ and carbonyl stretching at 1681-1653 $\mathrm{cm}^{-1}$.

Sodium salt of acridone-4caboxylic acid 9 was prepared by cyclodehydration of $\mathrm{N}-(2-$ caboxyphenyl)anthranilic acid $\quad \mathbf{7}^{19}$ obtained through Jordan-Ullmann copper catalyzed condensation between 2-chlorobenzoic acid $\mathbf{5}$ and anthranilic acid 6 to give acridone-4carboxylic acid $\mathbf{8}^{19 \& 20}$ which was dissolved in sodium hydroxide solution to give sodium 9-oxo-9,10dihydroacridine-4-carboxylate $\quad \mathbf{9}^{19}$. Refluxing 4a-h with 9 in dry DMF yielded compounds 10a-h. IR spectra showed aliphatic $\mathrm{CH}_{2}$ stretching at about 2928, $2857 \mathrm{~cm}^{-1}$ and carbonyl stretching at about 1738, 1689, 1620 corresponding to ester, hydrazide and acridone $\mathrm{C}=\mathrm{O}$ respectively. ${ }^{1} \mathrm{H}$ NMR revealed a singlet at $\delta=5.11 \mathrm{ppm}$ attributed to $\mathrm{CH}_{2}$ protons in addition to multiplet at $\delta=7.08-8.59 \mathrm{ppm}$ corresponding to 12 aromatic protons and $\mathrm{NH}$ proton and singlet at $\delta$ $=10.36 \mathrm{ppm}$ and $11.6 \mathrm{ppm}$ corresponding to $\mathrm{NH}$ protons for compound 10a.

Chlorination of 10a using thionyl chloride and few drops of DMF (as catalyst $^{22-26}$ gave phenylhydrazinocarbonylmethyl 9-chloroacridine-4carboxylate $\mathbf{1 1}$ which was added to an equimolar amount of aromatic amines or sulfonamides dissolved in DMF acidified with hydrochloric acid and stirred to give compound 12a-k. IR spectra showed stretching of aliphatic $\mathrm{CH}_{2}$ and carbonyl function group at about 2917, 2860 and 1692, $1668 \mathrm{~cm}^{-}$ ${ }^{1}$ respectively. ${ }^{1} \mathrm{H}$ NMR exhibited the appearance of a singlet at $\delta=5.14$ ppm corresponding to $\mathrm{CH}_{2}$ protons for compounds $12 \mathrm{a}$ and $\mathbf{1 2 k}$.

Furthermore, reaction of sodium 9-oxo-9,10-dihydroacridine-4-carboxylate 9 and mono chloroacetic acid afforded 13. IR spectra showed stretching of aliphatic $\mathrm{CH}_{2}$ at 2926 and $2850 \mathrm{~cm}^{-1}$ in addition to carbonyl stretching at 1722, 1688 and $1622 \mathrm{~cm}^{-}$ ${ }^{1}$ of the acid, ester and acridone. ${ }^{1} \mathrm{H}$ NMR revealed a singlet at $\delta=4.93$ ppm for $\mathrm{CH}_{2}$ protons.

Chlorination of 9-Oxo-9,10dihydroacridine-4-carbonyloxyacetic acid 13 using thionyl chloride and 
few drops of DMF (as catalyst) ${ }^{10,20 \& 27}$ provided 2-chloro-2-oxoethyl 9chloroacridine-4-carboxylate 14. The newly introduced chlorine atoms were either substituted simultaneously in one step with 2 moles of appropriate aromatic amines or sulfonamides ${ }^{27}$ to give compounds $\mathbf{1 5}$ or the acid chloride was preferentially substituted with equimolar amount of amine at low temperature under mild basic anhydrous conditions to yield intermediate phenylcarbamoylmethyl 9-chloroacridine-4-carboxylate $\mathbf{1 6}$ which was further reacted with equimolar amount of different aromatic amines or sulfonamides under mild acidic conditions ${ }^{20 \& 27}$ to give compound 17 .

IR spectra showed $\mathrm{CH}_{2}$ stretching at about 2930, $2836 \mathrm{~cm}^{-1}$ and carbonyl stretching at about 1742 , $1667 \mathrm{~cm}^{-1}$. ${ }^{1} \mathrm{H}$ NMR spectra revealed a singlet at $\delta=4.95 \mathrm{ppm}$ for $\mathrm{CH}_{2}$ protons in addition to multiplet at $\delta$ $=7.28-8.59 \mathrm{ppm}$ assigned to 17 aromatic protons and singlet at $\delta$ $=11.44 \mathrm{ppm}$ and $11.48 \mathrm{ppm}$<smiles>[R]c1ccc(NNNC(=O)COC(=O)c2cccc3c(=O)c4ccccc4[nH]c23)cc1</smiles>

A

1- Compounds A showed no activity.

2- Compounds B showed good activity when $R_{1}$ and $R_{2}$ are sulfamoyl moiety or when only $\mathrm{R}_{2}$ corresponding to $\mathrm{NH}$ protons for compound 15a.

IR spectra showed $\mathrm{CH}_{2}$ stretching at about 2936, $2870 \mathrm{~cm}^{-1}$ and carbonyl stretching at about 1737 , $1689 \mathrm{~cm}^{-1}$. ${ }^{1} \mathrm{H}$ NMR spectra showed a singlet at $\delta=4.99 \mathrm{ppm}$ for $\mathrm{CH}_{2}$ protons in addition to multiplet at $\delta=$ 7.29-8.53 ppm attributed to 16 aromatic protons and $\mathrm{NH}_{2}$ protons and singlet at $\delta=10.95 \mathrm{ppm}$ and $11.97 \mathrm{ppm}$ corresponding to $\mathrm{NH}$ protons for compound $\mathbf{1 7 f}$.

Mass spectra of some representative compounds $10 \mathrm{a}, 10 \mathrm{~g}$, 12a, 12b, 15a, 15b, 17a and 17b were in compliance with their molecular weights.

\section{Antitumor screening}

The results showed that eight acridine derivatives $\mathbf{1 2 g}, \mathbf{1 2 h}, \mathbf{1 2 k}$, 15g, 15h, 17f, 17g, 17j exhibited good level of antitumor activity. The relationship between different substituents and activity is described as follow:<smiles>[R2]c1ccc(Nc2c3ccccc3nc3c(C(=O)OCC(N)=O)cccc23)cc1</smiles>

B $\mathrm{n}=1,2$

was sulfamoyl moiety. But, no activity when $R_{1}$ and $R_{2}$ were unsubstituted or when substitution was other than sulfamoyl moiety. 


\section{Conclusion}

Acridone derivatives 10 bearing 4substituted phenylhydrazinocarbonyl methyl 4-caboxylate at position 4 showed no antitumor activity although compound $10 \mathrm{~h}$ revealed comparable torsion angle $(10,4 \mathrm{a}, 4$, $\mathrm{CO})$ and torsion angle (10a, 8a, 9, 9a) compared to I and II which were reported to possess marked antitumor activity.

Introduction of sulfonamido or anilino or substituted anilino moiety to 4-substituted phenylhydrazinocarbonylmethyl acridine-4-caboxylate or 4-substituted phenylcarbamoylmethyl acridine-4-carboxylate at position 9 showed antitumor activity in case of compounds having sulfonamide substitution as shown by comparable torsion angle $(9,11,12$, 13) and torsion angle $(9 \mathrm{a}, 9,11,12)$ of compounds 12g, $15 \mathrm{~g}$ and $17 \mathrm{f}$ with that of compound IV indicating similar alignment of 9-anilino moiety. Therefore, we concluded that substitution with 9-anilino moiety carrying sulfamoyl group exhibits good antitumor activity.

\section{REFERENCES}

1- L. P. G. Wakelin, G. J. Atwell, G. W. Rewcastle and W. A. Denny, J. Med. Chem., 30, 855 (1987).

2- V. A. Bacherikov, T.-C. Chou, H.-J. Dong, X. Zhang, C.-H. Chen, Y.-W. Lin, T.-J. Tasi, R.Z. Lee, L. F. Liu and T.-L. Su, Bioorg. Med. Chem., 13, 3993 (2005).
3- V. A. Bacherikov, T.-C. Chou, H.-J. Dong, C.-H. Chen, Y.-W. Lin, T.-J. Tasi and T.-L. Su, Bioorg. Med. Chem. Lett., 14, 4719 (2004).

4- P. Yang, Q. Yang and X. Qian, Tetrahedron, 61, 11895 (2005).

5- P. Yang, Q. Yang, X. Qian, L. Tong and X. Li, J. Photochem. Photobiol. B: Biol., 84, 221 (2006).

6- S.-S. Wang, Y.-J. Lee, S.-C. Hsu, H.-O. Chang, W.-K. Yin, L.-S. Chang and S.-Y. Chou, Bioorg. Med. Chem., 15, 735 (2007).

7- K. A. Olaussen, K. Dubrana, J. Domont, J. P. Spano, L. Sabatier, and J. C. Soria,, Crit. Rev. Oncol. Hematol., 57, 191 (2006).

8- C. Martins, M. Gunaratnam, J. Stuart, V. Makwana, O. Greciano, A. P. Reszka, L. R. Kelland and S. Neidle, Bioorg. Med. Chem. Lett., 17, 2293 (2007).

9- B. D. Palmer, G. W. Rewcastle, G. J. Atwell, B. C. Baguley and W. A. Denny, J. Med. Chem., 31, 707 (1988).

10- M. Y. Ebeid, S. M. El-Moghazy Aly, A. N. Mikhael and A. A. H. Eissa, Egypt. J. Pharm. Sci., 33, 293 (1992).

11- A. K. Todd, A. Adams, J. H. Thorpe, W. A. Denny, L. P. G. Wakelin and C. J. Gardin, J. Med. Chem., 42, 536 (1999).

12- H. A. Abu-Shady, H. Safwat and S. El-Moghazy, Egypt. J. Pharm. Sci., 19, 85 (1978). 
13- HyperChem Release 4 for windows, molecular modeling software, Serial No. 51910007596 (1994).

14- H. Seefried, Chem. Ber., 55, 1827 (1922).

15- J. B. Muenzen, L. P. Cerecedo and C. P. Sherwin, J. Biol. Chem., 67, 469 (1926). Through Chem. Abstr., 20, 1837, (1926).

16- B. S. Furniss, A. J. Hannaford, P. W. G. Smith and A. R. Tatchell "Vogel's Text Book of Practical Organic Chemistry", $5^{\text {th }}$ Ed., Longman Scientific and Technical, Longman Group UK Ltd, 1989, p. 960.

17- K. Itano, J. Pharm. Soc. Japan, 75, 441 (1955). Through Chem. Abstr., 50, 22552d, (1956).

18- T. Hidaka, T. Kawakami (Nippon Soda Co., Ltd., Japan). Jpn. Kokai Tokkyo Koho JP 2002 249,481 (Cl.C07C323/60) 6 sep, 2002, JP Appl. 2000/389,703, 22 Dec 2000. Through Chem. Abstr., 137, 224142h, (2002).

19- S. M. El-Moghazy Aly, Egypt. J. Pharm. Sci., 33, 527 (1992).
20- G. J. Atwell, B. F. Cain, B. C. Baguley, G. J. Finlay and W. A. Denny, J. Med. Chem., 27, 1481 (1984).

21- P. Skehan, R. Storeng, D. Scudiero, A. Monks, J. McMahon, D. Vistica, J. T. Warren, H. Bokesch, S. Kenney and M. R. Boyd, J. Natl. Cancer Inst., 82, 1107 (1990).

22- H. I. El-Subbagh, A. H. Abadi and H. A. Al-Khamees, Arch. Pharm. Pharm. Med. Chem.,330, 277 (1997).

23- B. F. Cain, R. N. Seelye and G. J. Atwell, J. Med. Chem., 17, 922 (1974).

24- G. J. Atwell, B. C. Baguley, G. J. Finlay, G. W. Rewcastle and W. A. Denny, ibid., 29, 1769 (1986).

25- W. A. Denny, G. J. Atwell and B. F. Cain, ibid., 22, 1453 (1979).

26- G. W. Rewcastle, G. J. Atwell, B. C. Baguley and W. A. Denny, ibid., 27, 1053 (1984).

27- S. M. El-Moghazy Aly, A. N. Mikhael, A. A. H. Eissa and M. Y. Ebeid, Egypt. J. Pharm. Sci., 34, 401 (1992). 